\title{
Interoperability of population-based patient registries
}

\author{
Nicholas Nicholson $^{a, *}$, Andrea Perego ${ }^{a}$ \\ ${ }^{a}$ European Commission, Joint Research Centre (JRC)
}

\section{ARTICLE INFO}

\section{Keywords:}

Population-based patient registries Interoperability

Federated semantic metadata registry framework

ISO/IEC 11179

Linked Open Data

\begin{abstract}
A B S T R ACT
Enabling full interoperability within and between population-based patient-registry domains would open up access to a rich and unique source of health data for secondary data usage. Previous attempts to tackle patient-registry interoperability have met with varying degrees of success, but a unifying solution remains elusive. The purpose of this paper is to show by practical example how a solution is attainable via the implementation of an existing framework based of the concept of federated, semantic metadata registries. One important feature motivating the use of this framework is that it can be implemented gradually and independently within each patient-registry domain. By employing linked open data principles, the framework extends the ISO/IEC 11179 standard to provide both syntactic and semantic interoperability of data elements with the means of specifying automated extraction scripts for retrieval of data from different registry content models. The examples provided address the domain of European population-based cancer registries to demonstrate the feasibility of the approach. One of the examples shows how quick gains are derivable by allowing retrieval of aggregated core data sets. The other examples show how aggregated full sets of data and record-level data might also be retrieved from each local registry. An infrastructure of patient-registry domains adhering to the principles of the framework would provide the semantic contexts and inter-linkage of data necessary for automated search and retrieval of registry data. It would thereby also lay the foundation for making registry data serviceable to artificial intelligence (AI) applications.
\end{abstract}

\section{Introduction}

Population-based patient registries (PBPRs) provide an important source of epidemiological data on account of the information they contain on specific diseases arising in populations in well-defined geo-spatial regions. The mapping of such information over regional and national boundaries highlights differences in epidemiological indicators that can lead to changes in healthcare practice [1]. Moreover, further linkage of registry data with other types of data, such as environmental, socioeconomic or dietary/lifestyle data covering the same populations can stimulate more specific and targeted research based on observed correlations.

The current limitations regarding access to patient registry data — even within a given patient domain - are widely apparent [11] and constitute a first major challenge without regard to the more complex one of linking data between heterogeneous registries covering different patient domains.

Previous attempts have been made at European Union (EU) level to address some of the underlying needs [5], particularly within the field of rare diseases in which the problem of interoperability is more acute on account of the widely different types of diseases classified within the same overall patient domain [30]. These efforts, however, remain largely focused within each specific patient-registry domain and though the ensuing solutions may ease access to Europeanharmonised data on a thematic disease level, the use of different metadata methodologies coupled with different data-

\footnotetext{
*Corresponding author

@icholas.nicholson@ec.europa.eu (N. Nicholson); andrea.perego@ec. europa.eu (A. Perego)

욜 https://ec.europa.eu/jrc/ (N. Nicholson);

https://ec.europa.eu/jrc/ (A. Perego)

ORCID(s): $0000-0003-1149-1426$ (N. Nicholson); $0000-0001-9300-2694$ (A. Perego)
}

registration and data-discovery mechanisms will still present a challenge for the inter-linkage of data between registry domains.

Moreover, without any over-arching strategy and overall coordination across patient registry domains, much effort will continue to be duplicated in terms of reinvention of solutions that have at their basis shared and common requirements. It would be worthwhile to find some way of uniting these efforts towards a common and scalable methodology. A practical approach would be one that did not require fundamental changes to already existing data representations or individual registry infrastructures but rather encouraged, wherever possible, mapping of local registry data structures to common metadata constructs and the means of retrieving data on the basis of those mappings. With a common framework in place, work within the specific patient-registry domains would converge more rapidly towards greater data interoperability with consequently greater scope for secondary data usage.

A framework with the potential of meeting all these requirements has already been demonstrated in the field of electronic health records (EHRs) [35]. The framework consists of a federated architecture of semantic ISO/IEC 11179 [23] metadata registries (MDRs) and provides an innovative way for accomplishing mapping of metadata across systems and semantic contexts using linked open data (LOD) principles.

The semantic MDR framework was initially proposed for enabling data inter-linkage between different EHR formats with a primary focus on mapping the individual common data elements (CDEs) to standard CDE models. The framework has also been successfully applied to secondary use of EHRs for post-marketing surveillance [36]. Combining the concepts of metadata description of ISO/IEC 11179 


\begin{tabular}{|c|c|}
\hline \multicolumn{2}{|l|}{ Abbreviations } \\
\hline $\begin{array}{l}\text { CDE Common Data Element } \\
\text { CR Cancer Registry } \\
\text { CS Classification Scheme }\end{array}$ & $\begin{array}{l}\text { METeOR Metadata Online Registry - Australia's Metadata Online } \\
\text { Repository for national metadata standards for the health, } \\
\text { aged care, community services, early childhood and housing } \\
\text { and homelessness sectors }\end{array}$ \\
\hline CSI Classification Scheme Item & OC Object Class \\
\hline DEC Data Element Concept & OWL Web Ontology Language \\
\hline ECIS European Cancer Information System & PARENT Cross Border PAtient REgistries iNiTiative \\
\hline EHR Electronic Health Record & PBPR Population-Based Patient Registry \\
\hline $\begin{array}{l}\text { ENCR European Network of Cancer Registries } \\
\text { EU European Union }\end{array}$ & $\begin{array}{l}\text { RD-Connect Integrated platform connecting databases, registries, } \\
\text { biobanks, and clinical bioinformatics for rare disease re- } \\
\text { search. }\end{array}$ \\
\hline $\begin{array}{l}\text { FAIR Findable, Accessible, Interoperable, Reusable - guiding prin- } \\
\text { ciples for making data available }\end{array}$ & $\begin{array}{l}\text { RDF Resource Description Framework } \\
\text { REST Representational State Transfer }\end{array}$ \\
\hline GDPR General Data Protection Regulation & RESTful Conforming to the REST architectural style \\
\hline ICD-10 International Classification of Diseases, 10th revision & RoR Registry of Registries \\
\hline $\begin{array}{l}\text { ICD-O-3 International Classification of Diseases for Oncology, 3rd } \\
\text { edition } \\
\text { IEC International Electrotechnical Commission }\end{array}$ & $\begin{array}{l}\text { SKOS Simple Knowledge Organization System } \\
\text { SPARQL Recursive acronym for SPARQL Protocol and RDF } \\
\quad \text { Query Language. }\end{array}$ \\
\hline ISO International Organization for Standardisation & SQL Structured Query Language \\
\hline LOD Linked Open Data & Turtle Terse RDF Triple Language \\
\hline MDR Metadata Registry & URI Uniform Resource Identifier \\
\hline
\end{tabular}

with the capacities of linked open data and semantic web technologies provides a powerful and highly adaptable datainteroperability framework. Indeed, the versatility of the model would make it amenable to any application requiring standardised data exchange and, arguably, the limitation of its applicability is constrained more by implementationbased decisions within the given domain than by the technological constraints themselves. This paper presents a proposal for an implementation of the framework to address the particular data interoperability issues in the field of PBPRs.

The remainder of this paper is organised as follows. Sections 2 and 3 provide an overview of ISO/IEC 11179 and of the federated semantic MDR framework, respectively. The main characteristics of population-based patient registries are outlined in Section 4, and Sections 5 and 6 illustrate how the proposed approach can be applied to, respectively, aggregated core and full data sets of European cancer registries. Section 7 discusses possible options to access individual PBPR records, while ensuring protection of personal data. Related work is discussed in Section 8. Section 9 assesses the significance of the approach illustrated in this paper, and Section 10 summarises the results, and presents the conclusions. Examples for creating the RDF representation are given in Appendix A.

\section{Brief overview of ISO/IEC 11179 metadata registries}

ISO/IEC 11179 [23] is a general description framework for data of any kind. It was prompted, amongst other things, by the need for standardised data-design procedures in order to ensure the emergence of data elements capable of sup- porting electronic data interchange [23]. According to the standard, metadata are viewed as "data about data in some context", where context can be considered as the set of circumstances, purposes, or perspectives relating to the data elements. In terms of the ISO/IEC 11179, a data element has therefore both semantic and representational components.

ISO/IEC 11179 distinguishes between the concept of a data element (Data Element Concept - DEC, describing the contextual semantics) and its representation (describing the permitted values a data element may use; the set of permitted values is called the Value Domain). The DEC further comprises two sub-components; the Object Class (OC), encapsulating the general underlying concept of the DEC; and the Property, a characteristic shared by all members of the OC (independent of any specific data-type). A data element is created when a specific DEC and a specific representation are associated together.

The Object Class, Property, and Value Domain provide searchable interfaces within the ISO metadata registry framework. For example, searching on a given Property would provide references to all Data Element Concept classes related to that Property and, through these, references are returned to all the associated Object Classes and Value Domains. An on-line application, METeOR [2], provides a good example of the means of searching for metadata elements within an ISO/IEC 11179 metadata registry.

By way of a specific example, METeOR defines one of its OCs as "Person with cancer". Associated with this OC are a number of Properties, one of which is "Primary site of cancer". The associated DEC is "Person with cancer primary site of cancer" which encapsulates the concept of a person with cancer having a primary site of the tumour. 
Interoperability of population-based patient registries

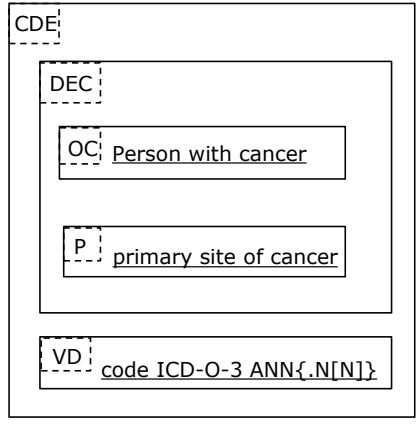

Figure 1: An example showing the construction of the CDE: "Person with cancer-primary site of cancer, code (ICD-O3) $\operatorname{ANN}\{. N[N]\}$ " from its constituent concepts according to ISO/IEC 11179, which include the Object Class (OC): "Person with cancer"; Property (P): "primary site of cancer"; and Value Domain (VD): "code (ICD-O-3) ANN $\{. N[N]\}$ ".

Since there exist a number of coding schemes for the conceptual domain denoting "tumour type", the "Person with cancer - primary site of cancer" DEC is free to associate with any of them (via assignment of different Value Domains), with each association providing a separate data element. Thus one data element is defined as: "Person with cancer-primary site of cancer, code (ICD-10-AM 7th edn) ANN $\{. \mathrm{N}[\mathrm{N}]\}$ " for descriptions of tumours according to the 7th edition of ICD-10 (International Classification of Diseases, 10th revision) [41], whereas another data element is defined as: "Person with cancer-primary site of cancer, code (ICD-O-3) ANN.N[N]" for descriptions of tumours according to ICD-O-3 (International Classification of Diseases for Cancer, 3rd edition) [22]. In METeOR, the fields "ANN $\{. \mathrm{N}[\mathrm{N}]\}$ " within the data elements' value domains refer to the format of the codes (one alphabetical character followed by two numeric characters with an optional decimal point followed by one or two numeric characters). The fact that the codes for these two schemes for classifying cancertype are expressed in exactly the same format underlines the need to make unambiguous distinction between the value domains, as supported by the ISO/IEC 11179 .

A further important principle of ISO/IEC 11179 concerns the use of classification schemes, which provide the means for developing metadata with enhanced semantic descriptions. Object Classes, Properties, Value Domains, DECs, and Data Elements are all classifiable components, and this aspect is an integral part of the philosophy underlying the federated semantic MDR framework.

By way of illustration, Figure 1 depicts the constituent concepts of the CDE "Person with cancer-primary site of cancer, code (ICD-O-3) ANN $\{. \mathrm{N}[\mathrm{N}]\} "$.

\section{Brief overview of the federated semantic MDR framework}

The federated semantic MDR framework enhances the capacity of the ISO/IEC 11179 metamodel by mapping its various constructs to associated OWL (Web Ontology Lan- guage) [38] classes and properties within an ontological representation of the metamodel. This mapping allows the different concepts of a data element to be described in RDF (Resource Description Framework) [24] and annotated where required via classification schemes to resources in other MDRs or to standard classification schemes / data dictionaries. In the federated semantic MDR framework each of these entities is identifiable by a URI (Uniform Resource Identifier) that may be dereferenced via the implementation of the necessary RESTful services. The framework also provides a semantic MDR service layer that allows federated queries to be made across the registered MDRs. The semantic MDR framework is described in greater detail in [35].

The ingenuity of the framework leads to a number of advantages; namely, that:

- it can be implemented gradually in a well-staged approach;

- it requires no fundamental change to the local data the underlying principle is to map local metadata to standard metadata descriptions or common dictionaries without enforcing compliance of metadata to any particular standard;

- it is scalable across many different patient-registry domains and can moreover be implemented in each domain independently of the other domains;

- not only are CDEs automatically registered and therefore findable, but they can be reused in an interoperable way via the semantic mapping descriptions to harmonised metadata standards.

Furthermore, via these mappings and their associated extraction specifications, local data elements described by otherwise non-standard CDEs are readily accessible. The semantic MDR framework therefore intrinsically supports all four of the FAIR (Findable, Accessible, Interoperable, Reusable) guiding principles for scientific data and stewardship [40].

\section{Brief overview of population-based patient registries}

PBPRs hold specific information of patients in a welldefined geographic area for which the population as a whole is known. At the highest level of data granularity, recordlevel data is considered sensitive because it holds identifiers, albeit pseudonymised, which can be used to trace back to an individual given the pseudonymisation key.

External user access to individuals' data is not usually required and anonymised, aggregated PBPR data provide a key data resource for epidemiological studies. Even for cases in which access to record-level data is required (for example in high-resolution studies), it is generally only necessary for the purpose of identifying the relevant cohorts of patients for the study, after which the personal identifiers are removed.

Data may be aggregated in a number of ways. One example is by age group whereby number of cases (incidence, 
mortality, etc.) is aggregated in predefined age-ranges. In case of rare occurrences of a specific type of disease, where the number of cases is low, data may also need to be aggregated across geographical area to avoid potential identification of individuals - this is particularly the situation encountered with rare-disease registries (RDRs).

Indicators (such as incidence, mortality, survival, and prevalence) provide the means of comparing the disease burden between different populations. These indicators are generally derived from a relatively narrow but harmonised subset of data - the core or common data set. Considerable effort is expended in ensuring the accuracy and comparability of the underlying data and therefore it is important to allow maximum re-use where possible.

It is oftentimes, however, not a straightforward matter even to find the core data sets. Moreover, the descriptions of the variables are not necessarily defined in rigorous and unambiguous terms. Just addressing these two aspects alone would bring an immediate breakthrough in the possibility of mapping different data sets along common fields of aggregation.

Beyond these more immediate returns, a rich source of untapped data resides in the aggregated full data sets. Although many of the data variables of the extended data sets may yet to be harmonised, describing them in terms of common metadata terminology would facilitate their incorporation into data analyses as well as their eventual mapping to harmonised variables once available.

Thus, the usefulness of PBPR data in terms of aggregated core data sets (for general comparison at population level), aggregated full data sets (for more specific comparison at population level), and record-level data (for highresolution studies) leads automatically to three fundamental use cases (elaborated in Sections 5, 6, and 7).

The use case for accessing record-level data is technically the most challenging since it requires access-control measures based on stringent data-protection needs to data held behind firewalls. The data-linkage mechanisms are in any case similar to the other use cases.

\section{Aggregated core data set of European cancer registries}

The aggregated core data set of the European Network of Cancer Registries (ENCR) consists of five main variables: indicator type (incidence/mortality), sex at birth, cancer-site, historical year, and number of cases broken down into fiveyear age ranges. Within a cancer registry's (CR) tabularised aggregated data set, these variables equate to the column names (c.f. Figure 2).

Currently this data is accessible from the European Cancer Information System (ECIS) [18], but requires some effort to extract in its entirety. Furthermore, the metadata is described in different places $[15,26]$, and not in machinereadable terms, nor do the majority of variables link to more generic metadata terms thereby rendering cross-linkage of data difficult between different PBPR domains.
In order to make the aggregated data set accessible via the semantic MDR framework, it is best transcribed in RDF, and maintained in a triple store. RDF provides a standard model for data interchange on the Web and allows structured and semi-structured data to be shared across different applications.

To transcribe the CR aggregated data in terms of RDF, the column names translate to the predicates of the RDF triples, with the row identifier (or primary key) forming the subject and the values of the predicates forming the objects.

The process is illustrated in the figures following. Figure 2 shows the header and one row of the aggregated data set corresponding to one CR (information taken from ECIS). It shows, within that particular CR, the number of incident male lung cancer cases in the associated age brackets per 100,000 head of male population in 2009.

The first step is to pivot the table of Figure 2 such that the individual age-brackets become data entities under a single "Age Group" column, as illustrated in the table in Figure 3. A primary key is also associated with each of the resulting rows. The first part of the key retains information of the original row number and the second part is the pivoted row number (associated with the corresponding age-bracket). The transcription of the pivoted table into RDF is shown in Figure 4 for the row identified by the row ID primary key "112 " (age bracket 55-59 of the original data). It consists of a number of RDF triples with the subject representing the row ID primary key, the predicate representing the column name, and the object the associated data value. The predicates have been given in terms of possible metadata conforming to the ISO/IEC 11179 model. For example, the predicate encr: personWithCancer_TumourPrimarySite_TumourCodeECISv1 is the URI associated with the CDE similar to that described earlier. In this CDE however, the Value Domain "TumourCodeECISv1" would have the list of possible values as defined currently by ECIS [15].

The data entry corresponding to the number of incident, male (at birth), lung cancer cases within a given CR for the year 2009, aggregated in the age group identifier 12 (55-59 years old) thus comprises six triples. Each triple has the same subject given by the common primary key value "112 ", the leading digit of which represents the row number of the original data. The predicates and the objects (apart from the xsd prefixes) point to currently fictitious URIs associated with specific values of the respective Value Domains' data-format attributes. Since most of the metadata types are generic to all PBPRs, they are best defined and maintained within a generic-PBPR semantic MDR that could then be referenced by different PBPR domains.

A tentative full RDF definition of the subjects, predicates, and objects used in this PBPR example is illustrated in Section A, to which we refer the reader for more details.

ISO/IEC 11179 defines a data element as a "unit of data that is considered in context to be indivisible", where context is defined as "the circumstance, purpose and perspective under which an object is defined or used" [23]. The standard provides the example of a telephone number that 


\begin{tabular}{|c|c|c|c|c|c|c|c|c|c|c|c|c|c|c|c|c|c|c|c|c|c|}
\hline $\begin{array}{l}\frac{1}{+} \\
\stackrel{0}{0} \\
\stackrel{\underline{0}}{0} \\
\underline{\underline{c}}\end{array}$ & $\stackrel{\times}{\stackrel{\times}{\sim}}$ & $\begin{array}{l}\overline{\bar{d}} \\
\stackrel{\bar{C}}{\tilde{U}}\end{array}$ & $\stackrel{\bar{\varpi}}{\stackrel{\varpi}{\nu}}$ & ণ্ & ํㅗ & $\begin{array}{l}\stackrel{+}{+} \\
\text { 하 }\end{array}$ & $\begin{array}{l}\stackrel{9}{\overrightarrow{1}} \\
\stackrel{\text { ம }}{-}\end{array}$ & $\begin{array}{l}\stackrel{\sim}{\sim} \\
\text { ㅇ }\end{array}$ & 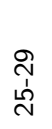 & $\begin{array}{l}\text { ले } \\
\text { dे }\end{array}$ & $\begin{array}{l}\text { ले } \\
\text { மे }\end{array}$ & $\begin{array}{l}\text { \& } \\
\text { ơ }\end{array}$ & $\begin{array}{l}\text { g } \\
\text { மे } \\
\text { ம) }\end{array}$ & $\begin{array}{l}\text { 華 } \\
\text { ○े }\end{array}$ & 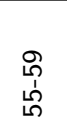 & \begin{tabular}{l}
\multirow{0}{0}{} \\
0 \\
0 \\
8
\end{tabular} & $\begin{array}{l}80 \\
01 \\
1 \\
0\end{array}$ & 永 & $\begin{array}{l}\stackrel{9}{i} \\
\stackrel{1}{R}\end{array}$ & 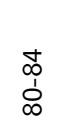 & $\underset{\infty}{+}$ \\
\hline 1 & $M$ & Lung & 2009 & 0 & 0 & 0 & 0 & 0 & 0 & 6 & 0 & 3 & 34 & 51 & 120 & 148 & 297 & 367 & 504 & 565 & 598 \\
\hline
\end{tabular}

Figure 2: Example of a row of a CR's aggregated core data set.

\begin{tabular}{|c|c|c|c|c|c|c|}
\hline $\begin{array}{l}0 \\
\frac{3}{0} \\
\simeq\end{array}$ & 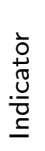 & $\stackrel{\times}{\stackrel{凶}{\omega}}$ & 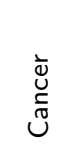 & $\stackrel{\bar{\pi}}{\stackrel{\bar{\nu}}{\nu}}$ & 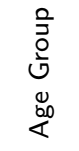 & $\begin{array}{l}\mathscr{J} \\
\stackrel{⿹}{J}\end{array}$ \\
\hline $1-1$ & I & $M$ & Lung & 2009 & $0-4$ & 0 \\
\hline $1-2$ & 1 & $M$ & Lung & 2009 & $5-9$ & 0 \\
\hline $1-3$ & 1 & $\mathrm{M}$ & Lung & 2009 & $10-14$ & 0 \\
\hline $1-4$ & 1 & M & Lung & 2009 & $15-19$ & 0 \\
\hline $1-5$ & 1 & $\mathrm{M}$ & Lung & 2009 & $20-24$ & 0 \\
\hline $1-6$ & 1 & $M$ & Lung & 2009 & $25-29$ & 0 \\
\hline $1-7$ & 1 & $\mathrm{M}$ & Lung & 2009 & 30-34 & 6 \\
\hline $1-8$ & 1 & M & Lung & 2009 & 35-39 & 0 \\
\hline $1-9$ & 1 & $\mathrm{M}$ & Lung & 2009 & $40-44$ & 3 \\
\hline $1-10$ & 1 & $\mathrm{M}$ & Lung & 2009 & $45-49$ & 34 \\
\hline $1-11$ & I & $\mathrm{M}$ & Lung & 2009 & 50-54 & 51 \\
\hline $1-12$ & I & M & Lung & 2009 & 55-59 & 120 \\
\hline $1-13$ & I & $\mathrm{M}$ & Lung & 2009 & $60-64$ & 148 \\
\hline $1-14$ & 1 & $\mathrm{M}$ & Lung & 2009 & $65-69$ & 297 \\
\hline $1-15$ & I & $\mathrm{M}$ & Lung & 2009 & $70-74$ & 367 \\
\hline $1-16$ & 1 & $M$ & Lung & 2009 & $75-79$ & 504 \\
\hline $1-17$ & 1 & M & Lung & 2009 & $80-85$ & 565 \\
\hline $1-18$ & 1 & $M$ & Lung & 2009 & $85+$ & 598 \\
\hline
\end{tabular}

Figure 3: Expanded representation of the row in Figure 2. The table includes a row for each of the age groups in Figure 2 , which are specified as values of a specific column ("Age Groups").

may be considered indivisible in one context but divisible in another (where telephone numbers need to be divided into country code, area code, and local number). To all intents and purposes, the core data set can be considered indivisible for the purpose of making it searchable and accessible as a whole. Not only are the core data sets standardised and harmonised within any given PBPR domain, but an individual field within the aggregated data set is not meaningful without explicit reference to the values of all the other fields in the same row. The way in which the core data set will eventually be used forms a higher-level context outside our immediate concern and since our interest is in allowing access to and retrieval of the core data sets as a whole within each PBPR domain, we specifically define a CDE to represent a registry's aggregated core data set. This marks a slight departure from the aim of [35] where the purpose was to extract the value of specific CDEs associated with the EHRs of specific patients. The alternatives (none of which are preclusive to the above) would be: (a) to specify CDEs representing each individual record within the aggregated core data set, e.g. the number of incident, male (at birth), lung cancer cases aggregated within the age range 55-59 years diagnosed in the year 2016. This however would require a major initial outlay of effort in defining the whole set of CDEs (for each individual

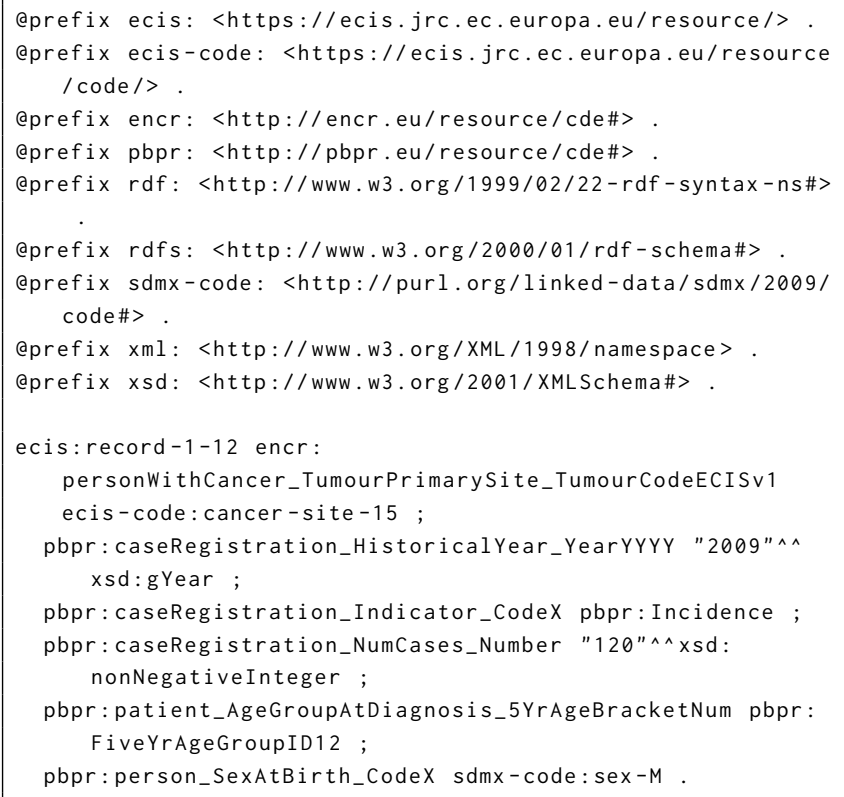

Figure 4: Example of a data entry in a CR's aggregated core data set in RDF, using the Turtle [32] serialisation, corresponding to the row with ID 1-12 in Figure 3.

tumour type, age bracket, indicator type, sex, and year) as well as complicate the task of reconstituting the entire aggregated core data set for users requiring it; or (b) to provide a user interface for selecting the particular fields of interest within the data set and on the basis of these choices, to run a script on the data set to return the relevant result. This option also would require more initial effort, although such an interface would be useful also in accessing individual record data (discussed in Section 7).

Following a similar semantic-MDR, schematic representation to that provided in [35], Figure 5 illustrates the definition of a proposed common data element (CDE) for the ENCR aggregated core data set with semantic links through the LOD cloud.

The CDE is the association of an Object Class that represents the concept of a population-based ENCR registry with a Property referring to the feature of an aggregated core data set, and a Value Domain that specifies the form of the data element (RDF-formatted text).

The Object Class of the CDE is annotated with a concept of a population-based patient registry (via an association with a classification scheme item) through the SKOS (Simple Knowledge Organization System) [28] mapping property "broader" to indicate that a population-based ENCR 


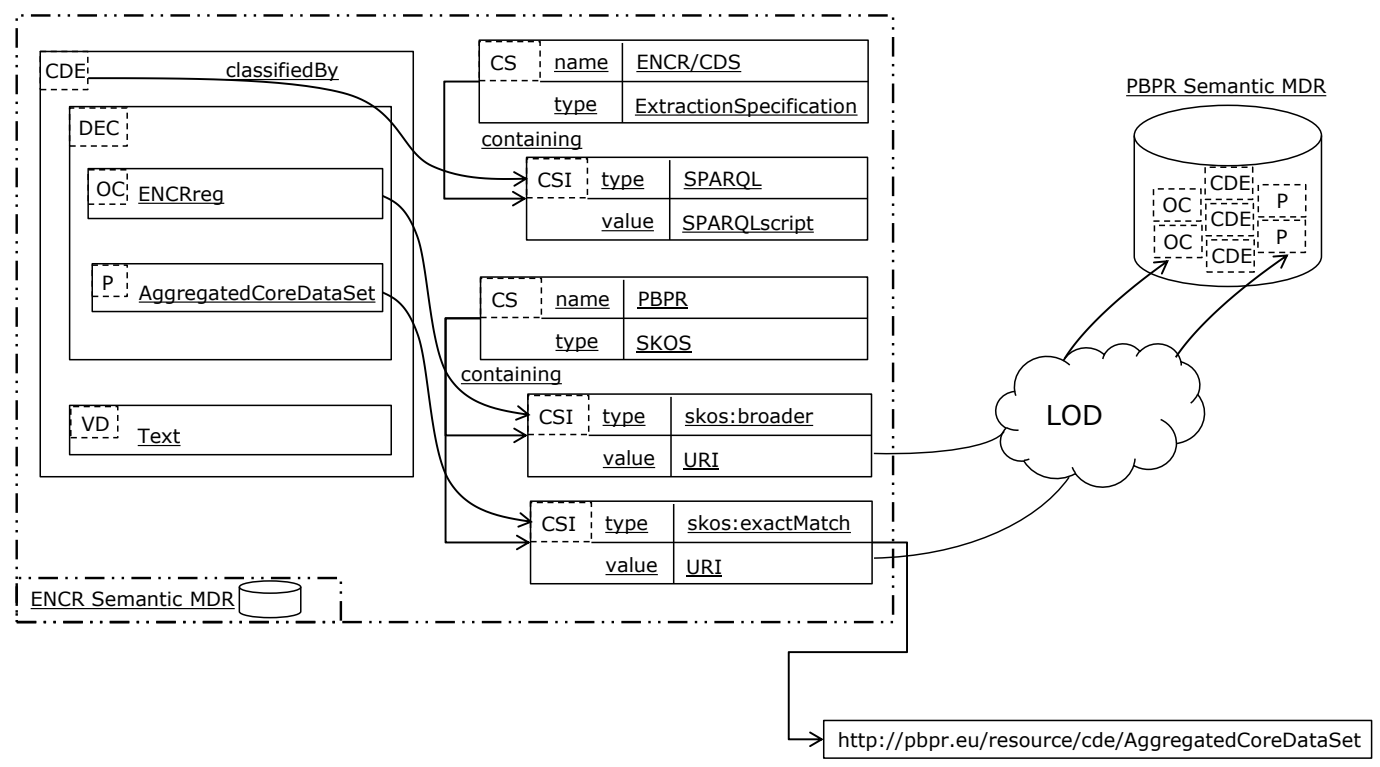

Figure 5: Semantic links of a CDE and its Property inside the ENCR semantic MDR. The Property $(P)$ is annotated through the SKOS [28] mapping property "exactMatch" to indicate that the CR core data set is an aggregated core data set of a populationbased patient registry. The Object Class (OC) is annotated through the SKOS mapping property "broader" to indicate that an ENCR registry is related to a population-based patient registry but is narrower in context. The CDE has an "Extraction Specification", which in this example is a SPARQL [39] script that is defined in Figure 6.

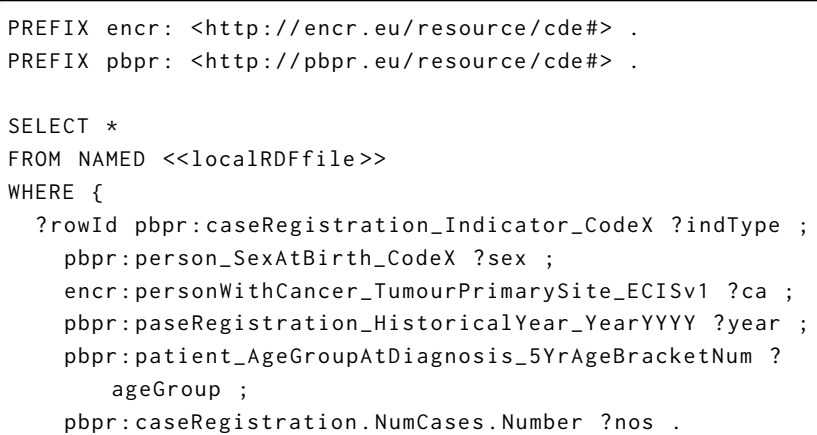

Figure 6: SPARQL script to retrieve an aggregate core data set from local CRs. The «localRDFfile» tag is a generic tag that is overwritten by the URI of the RDF graph containing the local data set. The latter is returned after searching the federated semantic MDR framework for links to the "PBencrRegistry.AggregatedCoreDataSet. Text" CDE.

registry is related, but narrower in context, to a PBPR.

The CDE also has an extraction specification specified with a SPARQL [39] script (described in Figure 6) that can be used to retrieve ENCR-conformant aggregated core data sets from local CR MDRs.

Executing the script in Figure 6 with the URI of a local registry's aggregated core data set would return all the records on a row-by-row basis with the individual data fields of each record aligned under the column names entitled by the predicates of the RDF triples. Since the latter are no less than the links to the CDE metadata describing the associated data fields, the user has all the information needed to ascer- tain in a relatively straightforward manner the full meaning of each record.

Two general and likely user scenarios, or sub use cases, can be considered. In the first scenario, a user is interested in accessing all the European cancer aggregated core data sets. To do this, the user would conduct a search on the CDE "ENCRreg.AggregatedCoreDataSet.Text" throughout the framework to find all the associated CDEs and their URIs. The SPARQL script of the CDE's extraction specification could then be run sequentially on each local semantic MDR's SPARQL endpoint (overwriting the «localRDFfile» tag with the URI of the RDF graph of the local MDR's CDE) to retrieve all the individual ENCR registries' aggregated core data sets.

In the second scenario, a user is interested in finding all the available population-based registries having aggregated core data sets. Searching through the semantic MDR framework on the Property "AggregatedCoreDataSet" would enable the user to find the URIs to all the aggregated core data sets linked to this Property for all PBPRs accessible in the framework. Refining the search on Object Class would then provide the means of classifying the URIs in terms of their specific domains (e.g. CR domain). Finally, the DEC together with the Value Domain define the CDE from which the extraction specification can be found for the specific registry domains. The user then has all the means by which to extract the data from all the local registries within each patient-registry domain.

Even achieving this result would in itself constitute a major milestone in the path towards data interoperability between registries and as well as open up for the first time the means of accessing data from all the various PBPR networks 


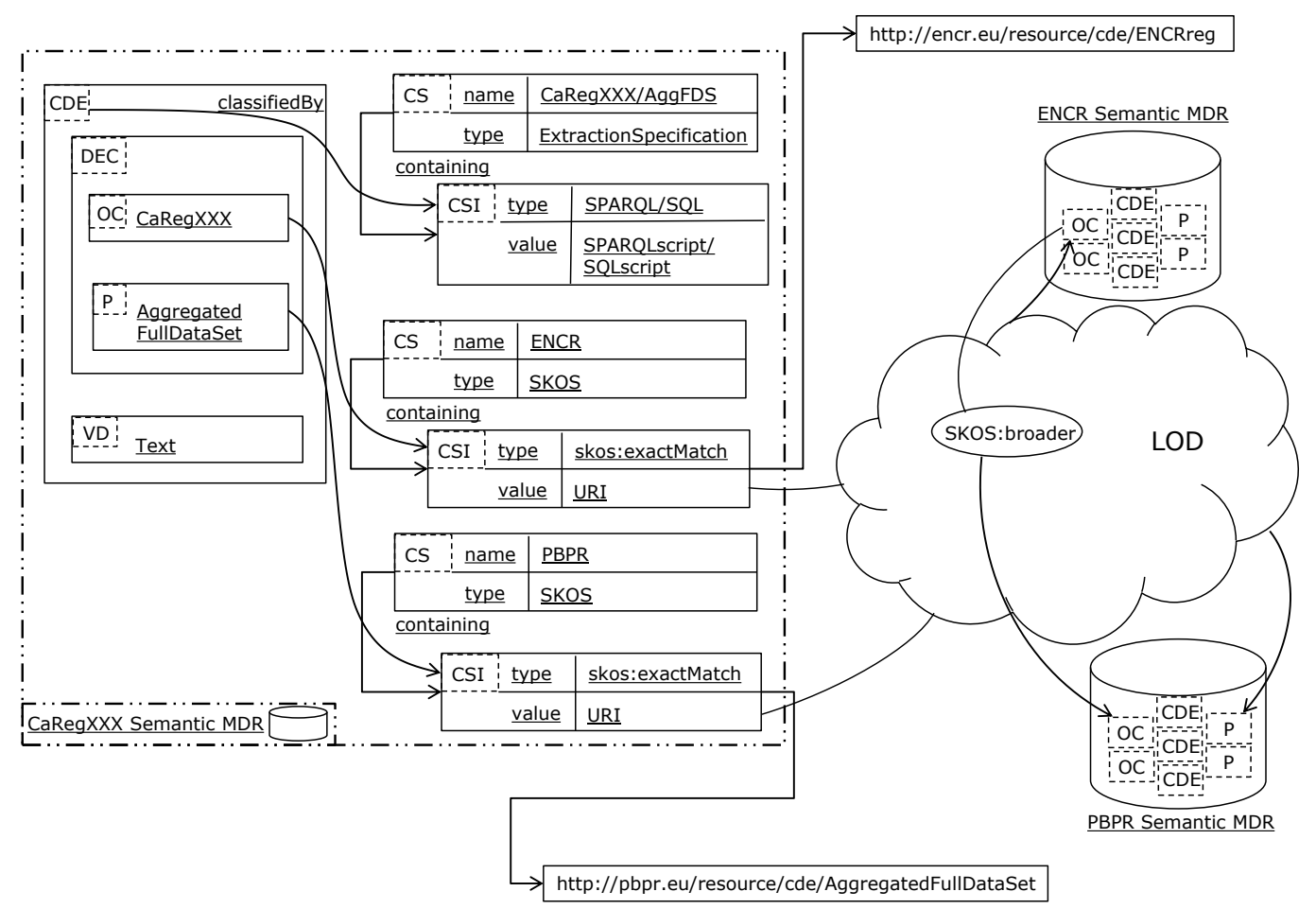

Figure 7: Semantic links of a CDE inside the local CR (CaRegXXX) semantic MDR. The Object Class (OC) is annotated through the SKOS mapping property "exactMatch" to indicate that the local registry is an ENCR registry-which is itself mapped to the PBPR OC via the SKOS mapping property "broader" (c.f. Figure 5). The Property (P) is also annotated through the SKOS mapping property "exactMatch" to indicate that the CR aggregated full data set is an aggregated European harmonized full data set. The CDE has an "Extraction Specification" that, dependent on local decisions, could be a SPARQL or SQL script to return the data set in a way similar to that described for the aggregated core data set.

without a major effort on behalf of the user. As an example, it would then be possible to look for potential correlations at population level between indicators relating to pancreatic cancer and type II diabetes in different geospatial regions and ascertain possible cohorts for further in high-resolution analyses.

\section{Aggregated full data sets of European cancer registries}

The core data sets, as useful as they are, hold only a fraction of the data potentially available. Access to the full set of a registry's data variables would not only provide users with much richer data sets but also serve, by wider use of the data, to accelerate the data-harmonisation process.

Due to the currently limited degree of harmonisation of the extended variable sets for many PBPRs, the CDEs of the non-harmonised variables and the extraction specifications for the full data sets would need to be provided and maintained by the local registries until such time as the variables became harmonised.

Figure 7 illustrates how the CDE for the full data set could be defined in the local MDR (CaRegXXX):

In the CDE of Figure 7, the Object Class is again used to refer to the concept of a population-based ENCR registry through the SKOS mapping property "exactMatch". The
Property refers to the concept of a population-based aggregated full data set also through the SKOS mapping property "exactMatch". The Value Domain specifies the form of the data element (which could be either RDF-formatted text or SQL-formatted text), and extraction specification for the aggregated full data set could accordingly be specified either in terms of SPARQL or SQL, depending on the local decision.

By searching on the Property "AggregatedFullDataSet" over the federated semantic MDR framework, the user would retrieve the URIs to all the full data sets from any associated population-based patient registry. The categorisation of patient-registry domain would again be determined from the OC. The DEC and Value Domain together define the CDE from which the extraction specification can be found. Running the extraction specification defined in the CDE of the local registry would retrieve the aggregated full data set stored in the local MDR.

It is perhaps important to add that whereas the semantic linkages of the CDEs would provide comprehensive descriptions of the CDEs themselves, this may still not be sufficient to provide users with a full understanding of data paradigm and the interdependency of the data variables themselves. For a higher-level view, it may be necessary to provide a data model or ontology describing the data domain. The description of such models can however be easily integrated into the semantic MDR framework and made accessible with the 
semantic descriptions of the associated Object Classes.

\section{7. "Tailor-requested" aggregation of data and access to record-level data of patient registries}

Access to all the data variables at record-level would allow the greatest use and value of patient-registry data, but requires explicit patient consent under the EU's recent general data protection regulation (GDPR) [21]. The record-level data is considered sensitive data even though in general it is pseudonymised through the recoding of the patient-identity field.

It should be emphasised that the ultimate reason why epidemiological studies require access to individuals' data is for the purpose of selecting the relevant cohorts of patients for testing a particular research hypothesis. Once the cohort is created, the analysis generally proceeds without further reference to individual patients. With this concept in mind, there are potentially two ways in which the data-sensitivity aspect of record-level data might be relaxed and allow data users more straightforward access to the underlying data:

1. By allowing users to specify the exact criteria for aggregating data. As an example, a user could ask the registry for the group of patients with survival less than a certain length of time suffering from a given cancer-type. As long as the number of corresponding patients were greater than a pre-defined minimum to prevent possible identification, the returned data set would be an aggregated measure and therefore essentially anonymous.

2. By providing users pseudonymised individual records with a minimal set of data fields. Minimising the number of data fields complicates the task of identifying a particular individual. This could be accomplished using a SPARQL/SQL front-end allowing users to specify search criteria based on a number of specific predicate/column names up to a permissible maximum number. Notwithstanding, appropriate measures should be in place to avoid successive calls on the same data being able to reconstruct the complete set of variables for any given record.

Data-access procedures for both these scenarios could in principle be automated since the results returned are arguably anonymised data. Requests of this nature could be constructed via a similar type of interface to that described in [36] albeit with extra functionality for handling service negotiation for data retrieval through a firewall.

\section{Related work}

Within the European Union (EU), a number of initiatives in context of the EU cross-border healthcare directive [20] have been undertaken to improve interoperability of patient registries.
One of the broader initiatives was the PARENT joint action [5], involving several EU Member States and partfunded by the European Commission's health programme 2008-2013. PARENT aimed both to rationalise and harmonise the development and governance of registries for the purpose of facilitating secondary data usage for research and public health. The action developed a set of methodological guidelines to help overcome commonly encountered challenges in the establishment, operation, and maintenance of registries [42].

PARENT also piloted a "registry of registries" (RoR) essentially foreseen as a web portal providing reliable and up-to-date information about European patient registries' metadata [31].

Within the Biobanking and Biomolecular Resources European Research Infrastructure (BBMRI-ERIC) [3], the MIABIS (Minimum Information About BIobank data Sharing) community standards [27] were developed for supporting biobank data interoperability. MIABIS provides standardised data elements for describing biobanks and as well as data on associated sample collections. The data elements are provided in XML [4], but do not conform to ISO/IEC 11179.

A non-exhaustive list of other past and present domainspecific initiatives include: RD-Connect [7], EU RD Platform [19] (rare-diseases domain); EUBirod [12] (diabetes domain); EUROCISS [14] (cardiovascular domain); EUROCOURSE [6], ECIS [18] (cancer domain); ECFSPR [10] (cystic-fibrosis domain). With the exception of RD-Connect, the CDEs developed within the domain-specific projects are described in PDF files [9, 13, 15, 16, 17, 26] and therefore cannot easily be queried or processed, nor are their semantic contexts provided according to the principles of linked open data. RD-Connect uses the COEUS framework [25] to build and provide the semantic context for rare-diseases registries. Given the sheer number of registries and variation in type of data repositories, COEUS facilitates the process towards semantic-knowledge federation. Whereas COEUS provides some of the functionality of the federated semantic MDR framework and eases the transition of traditional data storage mechanisms towards semantic web technologies, it would be unnecessarily restrictive to force it upon all registry domains. Furthermore, COEUS derives the predicates of the RDF triples from the column names of the underlying data sources and then maps them to the relevant predicates in a given ontology, wherein can lie potential inconsistencies as discussed in [34]. In contrast, the federated semantic MDR framework has these mappings already established within the local MDRs and linked at the different conceptual levels of a data element through the classification scheme associations, allowing greater flexibility in semantic searches. However, some of the shared similarities could potentially be used to provide the semantic mappings to access data elements between the frameworks. Full alignment would be possible by defining the CDEs in terms of ISO/IEC 11179 and then using the semantic MDR framework to link the CDEs to the associated semantic mappings created using 


\section{COEUS.}

\section{Discussion}

The intention of this work has been to show at a practical level how the implementation of the federated semantic MDR framework might greatly facilitate interoperability within and between population-based patient registry domains.

The extension of the framework however goes far beyond a single registry domain and provides the means of interlinking data from the whole patient-registry community. Indeed, such a framework could also incorporate clinical registries and thereby eventually automate the whole data-flow processes from the primary data sources to the populationbased patient registries and, conversely, provide links back to more comprehensive patient data when required (e.g. for high-resolution epidemiological research).

In consideration of the advantages and gains that the framework would provide, the steps to accomplish it are meritorious of the required effort. In summary, these steps would be:

1. Within the PBPR generic domain: to create a number of abstract ISO/IEC 11179 classes (in particular Object classes and Properties) that can be reutilised at specific registry domain level. Examples would include "Population-Based Patient Registry" as an Object Class, and Properties such as: "Aggregated Core Data Set", "Aggregated Full Data Set", "Indicator Type", "Sex At Birth", "Year", "Geolocation Code", etc. These would provide the hooks for finding all the associated CDEs across different PBPR domains

2. Within the specific patient-registry domain:

(a) to create the required set of CDE definitions according to ISO/IEC 11179. One example for the CR domain would be the CDE definition for "Cancer Type", as discussed earlier. Metadata at this level will already exist - at least for describing the variables used within the core data sets - but may not be available in machine-readable ways. In order to reduce the effort, the initial focus could be limited to the definition of the core data variables;

(b) to set up the semantic links (via the Classification Scheme Item of ISO/IEC 11179) in the manner described by the semantic MDR framework to any relevant standard terminology systems (providing data dictionaries, classification schemata, ontologies, etc.) to which the CDEs are related. Extraction specifications will also need to be provided for the aggregated core and full data sets in the manner described in this paper. The extraction specifications are used to extract the associated aggregated data sets from the local patient registries;

3. Within the local registry/central registry domain: to provide the aggregated core data sets in RDF format;
4. For all MDRs: to establish a RESTful interface enabling the services foreseen by the semantic MDR framework (namely, SPARQL endpoint, CDE endpoint, CDE search, Semantic links, and Extraction specification).

As an initial approach, if the central coordinating entities of the patient-registry domains store the aggregated core data sets as a type of proxy for the local registries, then the central semantic MDR of the different PBPR domains could service all the requests to retrieve the individual core data sets. However, to unlock the full power of the registry data, each individual local registry would also need to set up their own semantic MDR and RESTful interface in order to handle the requests on the local registry. As a result, all data sets would reside on the servers of the local patient registries without the need for any central-level repository; furthermore, automatic access to non-sensitive aggregated full variable-set data would then be possible.

In view of the fact that the implementation of these steps will share many commonalities between registries and also for ease of rolling out such a framework encompassing all population-based registries, it would be worthwhile to prototype the whole concept on one patient-registry domain and, in so doing, create an implementation manual for other domains to follow.

The CR domain would serve as a good starting point. The CR domain is well established and comprises over 200 individual registries. Currently the core data sets are collected centrally and thereafter cleaned and aggregated prior to being made available on the ECIS website [18]. The aim is eventually to eliminate the central data-collection process altogether, thereby avoiding extra overheads and delays as well as the need for retaining copies of data sets with all the consequent maintenance and data-integrity issues. One of the hurdles to overcome before this becomes a reality however concerns the data-validation operation. Data validation is necessary at the central level to ensure all the data sets conform to a similar degree of data quality such that they can more accurately be compared. Work is in progress to provide open-source data-validation software tools based on a standard ENCR data model [29], which would allow a federated approach also to the data-validation process. One acid test will be to ascertain if these tools in conjunction with the framework itself provide the necessary robustness to devolve the current prerequisite central processes to the local level. In any case, self-regulation strongly motivates conformance to standard procedures and such a process could well be encouraged via the allocation of data-quality stamps to distinguish between different degrees of quality of data sets. Where data is seen to be essential, more effort will be given to ensuring compliance to standard practices.

\section{Results and conclusions}

The federated semantic MDR framework provides a powerful and versatile solution for enabling interoperability of data across heterogeneous patient registries, essentially by 
mapping local metadata to standard metadata descriptions and linking their components semantically via knowledge organisation system ontologies and terminology systems without enforcing compliance to any one common data model. In a world where data has long been collected and managed with local contexts in mind, this is a critical aspect towards allowing secondary data usage without requiring fundamental changes to existing data sets or local datacollection practices.

One of the strengths of the framework lies in the fact that it is not a disruptive technology but rather provides the means, via the integration of a number of powerful tools and standards, to link data that would otherwise remain fragmented.

Whereas the implementation is not cost-neutral - a number of elements need to be established and thereafter maintained - and registries already contending with limited resources would undoubtedly require support, the following points must be borne in mind:

- Population-based patient registries contain valuable and important data stretching over many years. The value of the data may be gauged from all the previous initiatives and endeavours to make them interoperable. If a registry is established, it makes inherent sense to ensure the data it collects are interoperable with those of other registries to provide extra value;

- Not agreeing a common framework only postpones the problem of data inter-linkage to some future date - data inter-linkage will always depend on semantic description of the data and where this has not been considered at an early stage, it will have to be done later on and at potentially greater cost;

- Many on-going efforts focused on bringing interoperability across registries purely within a specific-patient domain are not resource-neutral and require considerable effort at all stages of the work. These resources could be re-directed;

- Agreeing a common approach brings economies of scale that can ease the burden on any one registry. Automated tools developed in the course of implementing the framework in one domain can be used to facilitate its implementation in other domains. The initial attention within an individual patient-registry domain need focus only on the critical aspects of metadata definition and semantic linkages;

- An infrastructure based on a framework that is designed for interoperability of EHRs means that interoperability between patient registries and EHRs is factored in from the start. The link to a patient identifier opens up a wealth of possibilities both for clinical processes and clinical research. On the one hand, it would ease the task of patient registries acquiring and validating data and on the other, any high-resolution study addressing a research question motivated from registry data would automatically have the link to many other related health details with which to select the pertinent patient cohort. The current difficulty in gaining access to this information is a cause of considerable effort, expense, and delay in such research;

- The framework can be implemented in degrees without breaking any of the underlying data processes. Metadata is not changed but rather mapped to standard metadata descriptions. Moreover, focusing initially on access to core data sets held centrally by each PBPR domain allows a quick win that can be rolled forward gradually to extend accessibility to other data variables;

- The framework provides a clear model and set of procedures for guiding the establishment of new patient registries and patient registry domains in order to make them compliant from the outset and thereby save future effort in making them interoperable;

- The issues raised within the PARENT guidelines [42] relating to registry data re-use (e.g. data compatibility and comparability; data exchange; mapping of classification codes; and data semantics) are all addressed by the functionality provided by the federated semantic MDR framework. In particular, metadata will be described in a formal manner, removing ambiguities and duplication of terms. Furthermore, it will be described in machine-readable terms and conform to the ISO/IEC 11179 metadata registry standard [23];

- Within the federated semantic MDR framework the need for any overarching registration function (such as PARENT's concept of an RoR) would be redundant all the metadata is already registered and linked in the framework and therefore can be browsed on a patientdomain basis using a tool similar to the one described in [36];

- The framework would eventually negate the need for any central collections of data for data-validation and data-cleaning needs, thereby avoiding further resourceintensive operations;

- With such a framework in place, attention and resources can be directed to developing user-interface tools for facilitating browsing, searching, marshalling, and fusing data retrieved from multiple data sources. These tools would serve as a front end to what would constitute a research infrastructure in its own right. Machine-readable metadata with their associated semantic descriptions and SKOS linkages to other resources would make the whole registry community amenable to the growing number of AI tools and applications and add the registry data sets to the growing pool of linked open-data resources. 


\section{A. Example of PBPR RDF representation}

The following sections illustrate a possible RDF implementation of the approach discussed in this paper by using the W3C RDF Data Cube vocabulary [8].

Although RDF Data Cube is modelled on SDMX [37], the standard for statistical data and metadata, it offers a general mechanism for representing tabular data that can be applied also in other domains. More precisely, each data item corresponds to an observation, consisting of a measurement of a given phenomenon along a given set of dimensions. E.g., the number of cancer cases in a given country in a given year, is represented as an observation where the number of cancer cases is the measurement, whereas country and year are dimensions.

The actual semantics of the used dimensions and measurements are defined as dimension and measure properties, respectively, along with their possible values. These definitions are also re-used to define the actual structure of the data set including an observation (referred to as "data structure definition").

Each data set is linked to the corresponding data structure definition, and each observation is linked to the corresponding data set. This approach has the advantage of associating an observation with provenance information (the data set) and with the semantics of each dimension and measurement used (defined in the data structure definition linked from the data set). Therefore, each observation carries and links to all the contextual information needed for its correct and unambiguous interpretation, even when it is shared and re-used independently or maintained in a shared database with other data sets.

The following sections provide a step-wise description of the definition, based on the RDF Data Cube vocabulary, of the example in Figure 4 from the aggregated core data set of the European Network of Cancer Registries (ENCR). For this purpose, the RDF definitions in the following sections make use of fictitious namespace URIs, with the exception of the one used for ICD-10 [41] cancer sites in Section A.5.4.

\section{A.1. Measure and dimension properties}

We start by defining the columns of PBPR records as dimension and measure properties.

In the PBPR record in Figure 4, the measurement corresponds to the number of cancer cases, which can be defined as shown in Listing 1.

\section{Listing 1: Measure property}

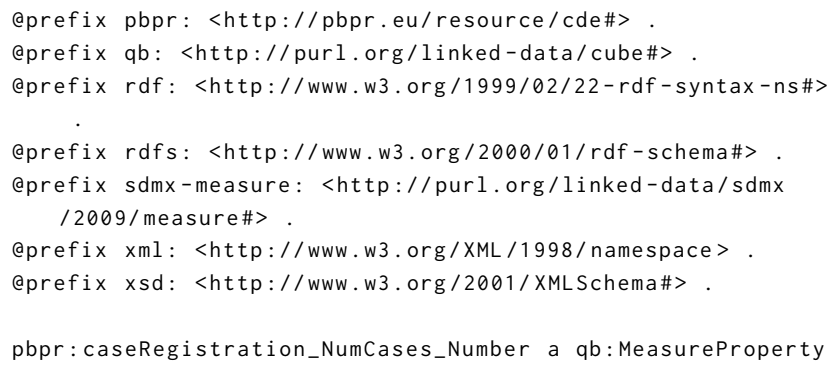

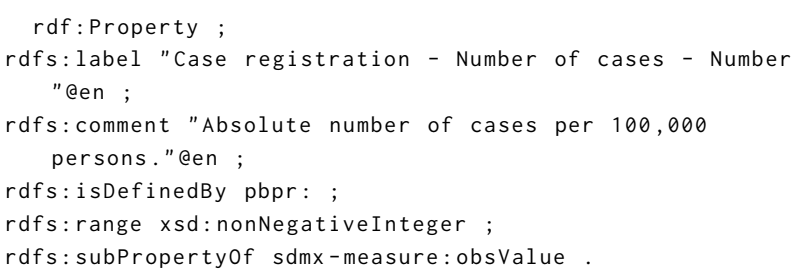

The rest of the columns denote the dimensions of the observation. Their RDF Data Cube definition is illustrated in Listing 2. It is worth noting that some of the defined dimension properties (i.e., those typed as qb: CodedProperty's) take as value not a literal, but an item from a given code list (specified via properties $q b$ : codelist and rdfs: range). The definition of the corresponding code lists is illustrated in Section A.5.

\section{Listing 2: Dimension properties}

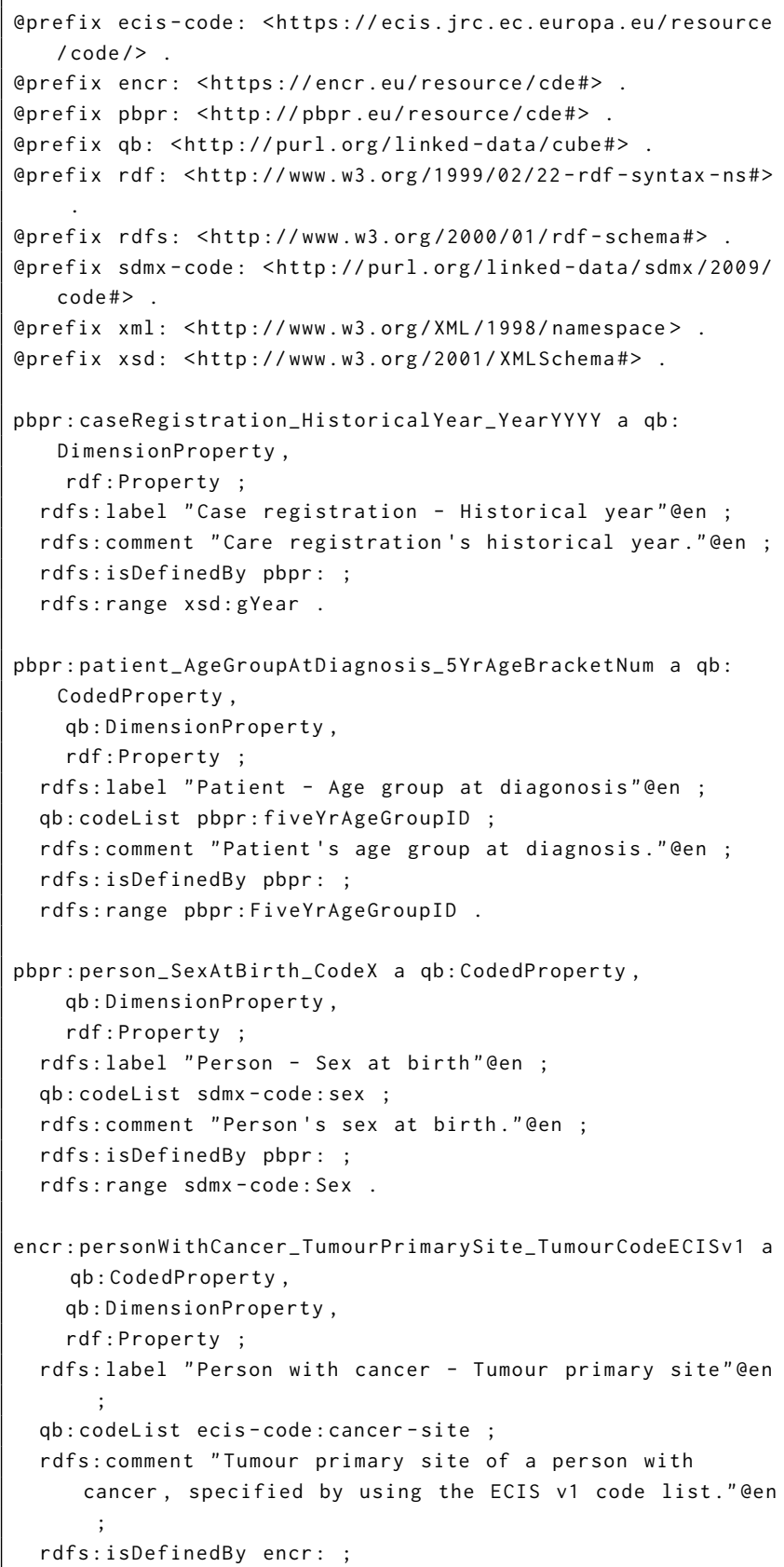


pbpr: caseRegistration_Indicator_CodeX a qb:CodedProperty, $q b$ : DimensionProperty,

rdf: Property ;

rdfs:label "Case registration - Indicator"@en ;

qb: codelist pbpr: caseRegistration_Indicator_CodeX ;

rdfs: comment "Case registration's indicator."@en ;

rdfs: isDefinedBy pbpr: ;

rdfs: range pbpr:CaseRegistration_Indicator_CodeX.

\section{A.2. Data structure definition}

The next step is defining the structure of the observations of the relevant data set, by specifying the corresponding dimension and measure properties.

The corresponding data structure definition is illustrated in Listing 3.

Listing 3: Data structure definition

\begin{tabular}{|l} 
@prefix ecis: <https://ecis.jrc.ec.europa.eu/resource/>. \\
@prefix encr: <https://encr.eu/resource/cde\#>. \\
@prefix pbpr: <http://pbpr.eu/resource/cde\#>. \\
@prefix qb: <http://purl.org/linked-data/cube\#>. \\
@prefix rdf: <http://www.w3.org/1999/02/22-rdf-syntax-ns\#> \\
@prefix rdfs: <http://www.w3.org/2000/01/rdf-schema\#>. \\
@prefix xml:<http://www.w3.org/XML/1998/namespace> . \\
@prefix xsd: <http://www.w3.org/2001/XMLSchema\#>. \\
ecis:dsd-1 a qb:DataStructureDefinition ; \\
qb:component [ qb:dimension pbpr: \\
caseRegistration_HistoricalYear_YearYYYY ], \\
[ qb:dimension encr: \\
personWithCancer_TumourPrimarySite_TumourCodeECISv1 \\
], \\
[ qb:dimension pbpr: \\
patient_AgeGroupAtDiagnosis_5YrAgeBracketNum ], \\
[ qb: dimension pbpr:person_SexAtBirth_CodeX ], \\
[ qb:measure pbpr:caseRegistration_NumCases_Number ], \\
qb: dimension pbpr:caseRegistration_Indicator_CodeX ]
\end{tabular}

\section{A.3. Data set}

Listing 4 illustrates the RDF Data Cube definition of the aggregated core data set of the European Network of Cancer Registries (ENCR). As it can be seen, the data set description links to the data structure definition in Listing 3 via property $\mathrm{qb}$ : structure.

\section{Listing 4: Data set}

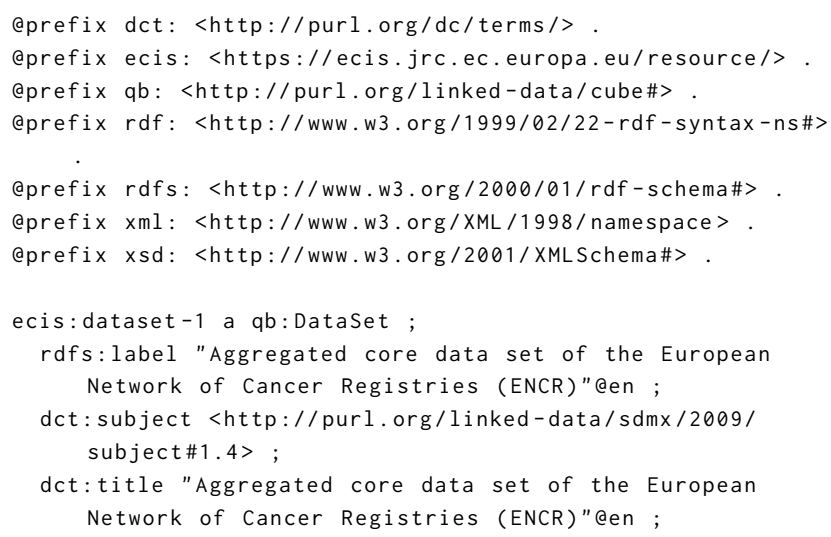

\section{A.4. Observations}

Listing 5 shows the RDF representation of the PBPR record in Figure 4, defined as an RDF Data Cube observation. The resulting definition is identical to the one in Figure 4, with the exception that it includes two additional statements-namely, one typing the record as a $\mathrm{qb}$ : Observation, and the other linking to the data set in Listing 4 via property $q b$ : dataSet.

\section{Listing 5: Observation}

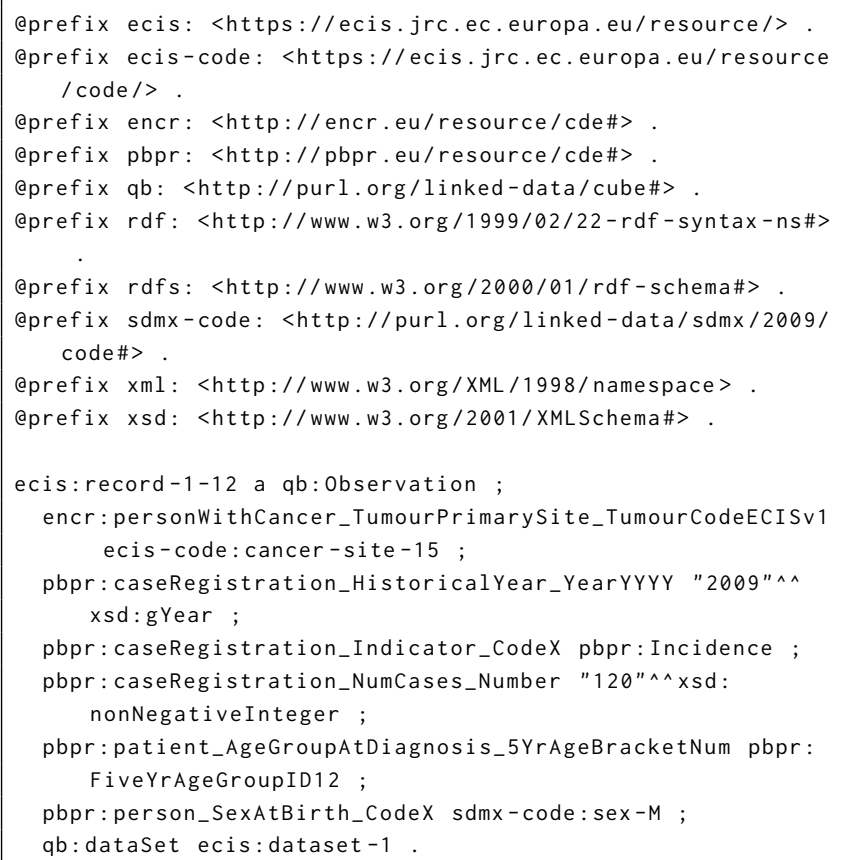

\section{A.5. Dimension properties code lists}

This section includes the RDF representation of the values for dimension properties, defined as code lists. Code lists are here defined by using SKOS [28], and following the approach used for the reference code lists of RDF Data Cube (see [8] and [33]). In particular:

- Each code list includes an RDF / OWL class, corresponding to the super-class of all the defined code list items, which can be used to specify the range of the dimension properties using them (see Section A.1).

- Each code list item is linked to the relevant code list by using both properties skos:inscheme and $\mathrm{qb}$ : codelist (see Section A.1).

\section{A.5.1. Sex}

The re-use of existing vocabularies is a best practice for Linked Data, as it facilitates interoperability. Based on this, for dimension property pbpr:person_SexAtBirth_CodeX it is possible to use the concepts defined in the relevant reference code list of RDF Data Cube (see [33]), supposing that these concepts are identical to the ones used in PBPRs. 


\section{A.5.2. Indicators}

This section includes the SKOS representation of case registration indicators used as values for dimension property pbpr: caseRegistration_Indicator_CodeX.

\section{Listing 6: Indicators}

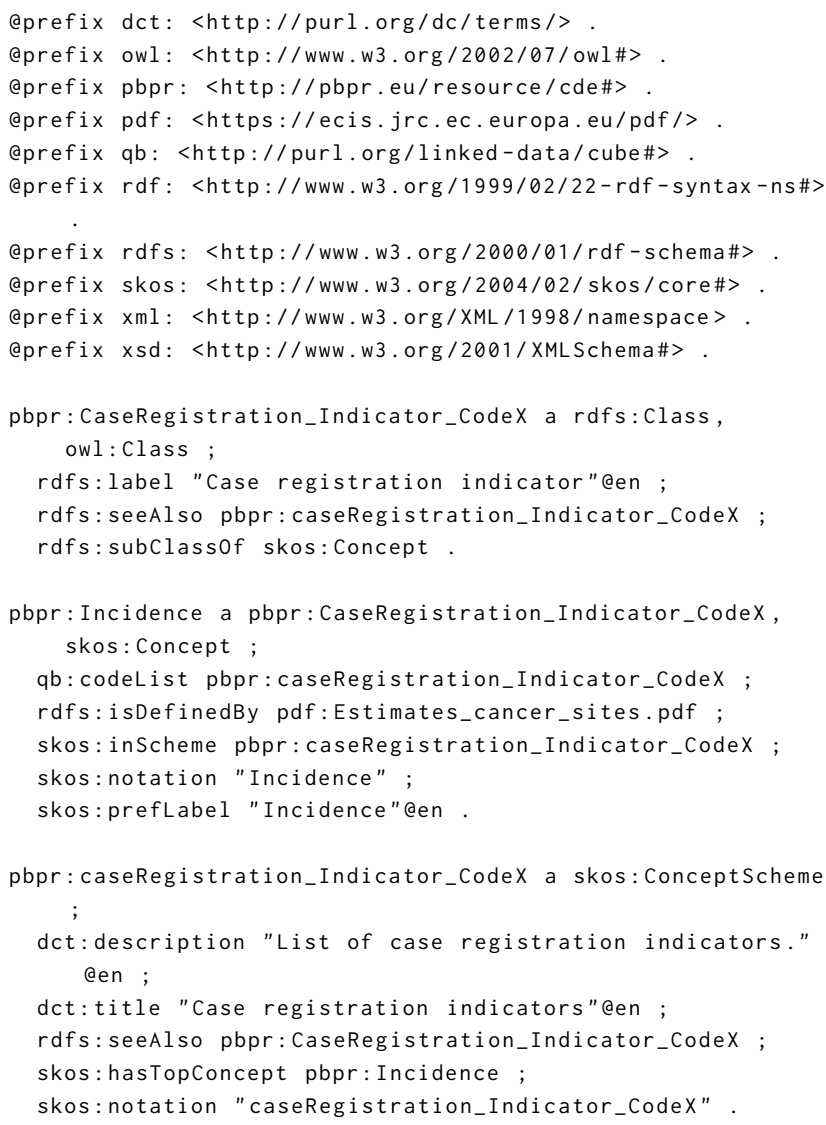

\section{A.5.3. Age groups}

This section includes the SKOS representation of 5-year age groups used as values for dimension property pbpr:patient AgeGroupAtDiagnosis_5YrAgeBracketNum.

\section{Listing 7: Age groups}

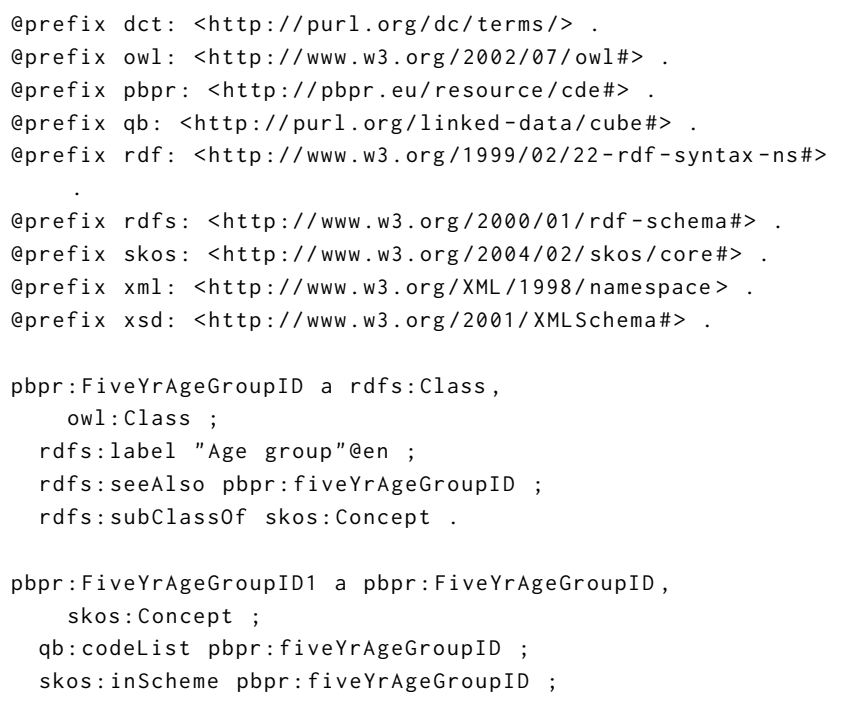

skos: notation "FiveYrAgeGroupID1" ; skos:prefLabel "Age group $0-4$ "@en.

pbpr:FiveYrAgeGroupID10 a pbpr:FiveYrAgeGroupID, skos: Concept :

qb: codelist pbpr: fiveYrAgeGroupID ; skos: inScheme pbpr:fiveYrAgeGroupID; skos: notation "FiveYrAgeGroupID10" ; skos:prefLabel "Age group 45-49"@en.

pbpr:FiveYrAgeGroupID11 a pbpr:FiveYrAgeGroupID, skos: Concept ;

qb: codelist pbpr:fiveYrAgeGroupID ; skos: inscheme pbpr:fiveYrAgeGroupID ; skos: notation "FiveYrAgeGroupID11" ; skos:prefLabel "Age group 50-54"@en.

pbpr:FiveYrAgeGroupID12 a pbpr:FiveYrAgeGroupID, skos: Concept ;

qb: codelist pbpr: fiveYrAgeGroupID ; skos:inScheme pbpr:fiveYrAgeGroupID ; skos: notation "FiveYrAgeGroupID12" ; skos:prefLabel "Age group 54-59"@en.

pbpr:FiveYrAgeGroupID13 a pbpr:FiveYrAgeGroupID, skos: Concept ;

qb: codelist pbpr:fiveYrAgeGroupID; skos: inScheme pbpr:fiveYrAgeGroupID ; skos: notation "FiveYrAgeGroupID13" ; skos:prefLabel "Age group 60-64"@en.

pbpr:FiveYrAgeGroupID14 a pbpr:FiveYrAgeGroupID, skos: Concept ;

qb: codelist pbpr:fiveYrAgeGroupID ; skos: inscheme pbpr:fiveYrAgeGroupID; skos: notation "FiveYrAgeGroupID14" ; skos:prefLabel "Age group 65-69"@en.

pbpr:FiveYrAgeGroupID15 a pbpr:FiveYrAgeGroupID, skos: Concept ;

qb: codelist pbpr:fiveYrAgeGroupID; skos: inscheme pbpr:fiveYrAgeGroupID; skos: notation "FiveYrAgeGroupID15". skos:prefLabel "Age group 70-74"@en.

pbpr:FiveYrAgeGroupID16 a pbpr:FiveYrAgeGroupID, skos: Concept

qb: codelist pbpr: fiveYrAgeGroupID; skos:inscheme pbpr:fiveYrAgeGroupID ; skos: notation "FiveYrAgeGroupID16" ; skos:prefLabel "Age group 75-79"@en.

pbpr:FiveYrAgeGroupID17 a pbpr:FiveYrAgeGroupID, skos: Concept;

qb: codeList pbpr:fiveYrAgeGroupID ; skos: inscheme pbpr:fiveYrAgeGroupID; skos: notation "FiveYrAgeGroupID17" ; skos:prefLabel "Age group 80-84"@en

pbpr:FiveYrAgeGroupID18 a pbpr: FiveYrAgeGroupID, skos: Concept ;

qb: codelist pbpr:fiveYrAgeGroupID; skos: inscheme pbpr:fiveYrAgeGroupID; skos: notation "FiveYrAgeGroupID18" ; skos: prefLabel "Age group 85+"@en.

pbpr:FiveYrAgeGroupID2 a pbpr:FiveYrAgeGroupID, skos: Concept

qb: codelist pbpr: fiveYrAgeGroupID; skos:inScheme pbpr:fiveYrAgeGroupID ; skos: notation "FiveYrAgeGroupID2" ; skos:prefLabel "Age group 5-9"@en. 
pbpr: FiveYrAgeGroupID3 a pbpr:FiveYrAgeGroupID, skos: Concept ;

qb: codelist pbpr:fiveYrAgeGroupID ;

skos: inscheme pbpr:fiveYrAgeGroupID;

skos: notation "FiveYrAgeGroupID3"

skos:prefLabel "Age group 10-4"@en.

pbpr:FiveYrAgeGroupID4 a pbpr:FiveYrAgeGroupID, skos: Concept ;

qb: codelist pbpr:fiveYrAgeGroupID ;

skos: inscheme pbpr:fiveYrAgeGroupID;

skos: notation "FiveYrAgeGroupID4" ;

skos:prefLabel "Age group 15-19"@en.

pbpr:FiveYrAgeGroupID5 a pbpr:FiveYrAgeGroupID, skos: Concept :

qb: codelist pbpr:fiveYrAgeGroupID ;

skos:inscheme pbpr:fiveYrAgeGroupID;

skos: notation "FiveYrAgeGroupID5" ;

skos:prefLabel "Age group 20-24"@en.

pbpr:FiveYrAgeGroupID6 a pbpr:FiveYrAgeGroupID, skos: Concept ;

qb: codelist pbpr:fiveYrAgeGroupID ;

skos: inscheme pbpr:fiveYrAgeGroupID;

skos: notation "FiveYrAgeGroupID6" ;

skos:prefLabel "Age group 25-29"@en.

pbpr:FiveYrAgeGroupID7 a pbpr:FiveYrAgeGroupID, skos: Concept ;

qb: codelist pbpr: fiveYrAgeGroupID ;

skos: inscheme pbpr:fiveYrAgeGroupID;

skos: notation "FiveYrAgeGroupID7" ;

skos:prefLabel "Age group 30-34"@en.

pbpr:FiveYrAgeGroupID8 a pbpr:FiveYrAgeGroupID, skos: Concept ;

qb: codelist pbpr:fiveYrAgeGroupID;

skos:inscheme pbpr:fiveYrAgeGroupID ;

skos: notation "FiveYrAgeGroupID8" ;

skos:prefLabel "Age group 35-39"@en.

pbpr:FiveYrAgeGroupID9 a pbpr:FiveYrAgeGroupID, skos: Concept ;

qb: codelist pbpr:fiveYrAgeGroupID ;

skos:inscheme pbpr:fiveYrAgeGroupID ;

skos: notation "FiveYrAgeGroupID9" ;

skos:prefLabel "Age group 40-44"@en.

pbpr:fiveYrAgeGroupID a skos: ConceptScheme; dct:description "List of age groups."@en ; dct:title "Age groups"@en ;

rdfs: seeAlso pbpr:FiveYrAgeGroupID; skos: hasTopConcept pbpr:FiveYrAgeGroupID1, pbpr: FiveYrAgeGroupID10, pbpr: FiveYrAgeGroupID11, pbpr: FiveYrAgeGroupID12, pbpr: FiveYrAgeGroupID13, pbpr: FiveYrAgeGroupID14, pbpr: FiveYrAgeGroupID15, pbpr: FiveYrAgeGroupID16, pbpr: FiveYrAgeGroupID17, pbpr: FiveYrAgeGroupID18, pbpr: FiveYrAgeGroupID2, pbpr: FiveYrAgeGroupID3, pbpr: FiveYrAgeGroupID4, pbpr: FiveYrAgeGroupID5, pbpr: FiveYrAgeGroupID6, pbpr: FiveYrAgeGroupID7, pbpr: FiveYrAgeGroupID8, pbpr: FiveYrAgeGroupID9

\section{A.5.4. ECIS cancer sites}

This section includes the SKOS representation of the ECIS code list for cancer sites, used as values for property encr: personWithCancer_TumourPrimarySite_TumourCodeECISv1. The definition of each cancer site in the code list includes also its mapping to the corresponding one in ICD-10 [41], via properties skos: exactMatch and skos: narrowMatch.

\section{Listing 8: Cancer sites}

aprefix dct. <http://purl.org/dc/terms/>

aprefix ecis-code: <https://ecis.jrc.ec.europa.eu/resource /code/>

aprefix icd10: <http://purl.bioontology.org/ontology/ICD10 l>.

aprefix owl: <http://www.w3.org/2002/07/owl\#>.

eprefix pdf: <https://ecis.jrc.ec.europa.eu/pdf/> .

eprefix qb: <http://purl.org/linked-data/cube\#>.

eprefix rdf: <http://www.w3.org/1999/02/22-rdf-syntax-ns\#>

aprefix rdfs: <http://www.w3.org/2000/01/rdf-schema\#>.

aprefix skos: <http://www.w3.org/2004/02/skos/core\#>.

aprefix xml: <http://www.w3.org/XML/1998/namespace>.

aprefix xsd: <http://www.w3.org/2001/XMLSchema\#>.

ecis-code: Cancersite a rdfs:Class,

owl: Class ;

rdfs:label "Cancer site"@en ;

rdfs: seeAlso ecis-code: cancer-site;

rdfs: subclassof skos: Concept.

ecis-code: cancer-site-1 a skos: Concept, ecis-code: CancerSite;

$q b$ : codelist ecis-code: cancer-site ;

rdfs: isDefinedBy pdf:Estimates_cancer_sites.pdf ;

skos:broader ecis-code:cancer-site-34;

skos: inscheme ecis-code:cancer-site ;

skos: narrowMatch icd10:c00,

icd10:c01,

icd10: $\mathrm{co2}$,

icd $10: \cos$,

icd $10: \mathrm{c} 04$,

icd10: $\operatorname{co5}$,

$\operatorname{icd} 10: \cos ;$

skos: notation "1";

skos:prefLabel "Lip, oral cavity"@en.

ecis-code: cancer-site-10 a skos: Concept,

ecis-code: Cancersite ;

qb: codelist ecis-code: cancer-site ;

rdfs: isDefinedBy pdf:Estimates_cancer_sites.pdf ;

skos:broader ecis-code: cancer-site-34;

skos: exactMatch icd10:c21;

skos: inscheme ecis-code: cancer-site;

skos: notation "10" ;

skos:prefLabel "Anus"@en.

ecis-code: cancer-site-11 a skos: Concept, ecis-code: CancerSite;

qb: codelist ecis-code: cancer-site ;

rdfs: isDefinedBy pdf:Estimates_cancer_sites.pdf ;

skos:broader ecis-code: cancer-site-34;

skos: exactMatch icd10:c22 ;

skos: inscheme ecis-code: cancer-site :

skos: notation "11";

skos: prefLabel "Liver"@en.

ecis-code: cancer-site-12 a skos: Concept,

ecis-code: Cancersite;

qb: codelist ecis-code: cancer-site ;

rdfs: isDefinedBy pdf:Estimates_cancer_sites.pdf ; 
skos:broader ecis-code: cancer-site-34; skos: inscheme ecis-code:cancer-site ; skos: narrowMatch icd10:C23,

icd10: 224 ;

skos: notation "12";

skos:prefLabel "Gallbladder"@en.

ecis-code: cancer-site-13 a skos: Concept, ecis-code: Cancersite :

$\mathrm{qb}$ : codelist ecis-code: cancer-site ;

rdfs: isDefinedBy pdf:Estimates_cancer_sites.pdf ;

skos:broader ecis-code: cancer-site-34;

skos: exactMatch icd10: 225 .

skos:inscheme ecis-code:cancer-site ;

skos: notation "13" ;

skos:prefLabel "Pancreas"@en.

ecis-code: cancer-site-14 a skos: Concept,

ecis-code: CancerSite ;

qb: codelist ecis-code: cancer-site ;

rdfs: isDefinedBy pdf:Estimates_cancer_sites.pdf ;

skos:broader ecis-code: cancer-site-34;

skos: exactMatch icd10:C32;

skos: inscheme ecis-code: cancer-site;

skos: notation "14" ;

skos: prefLabel "Larynx"@en.

ecis-code: cancer-site-15 a skos: Concept, ecis-code: CancerSite;

qb: codelist ecis-code: cancer-site ;

rdfs: isDefinedBy pdf:Estimates_cancer_sites.pdf ; skos:broader ecis-code:cancer-site-34;

skos:inscheme ecis-code:cancer-site ;

skos: narrowMatch icd10:C33,

icd10: $\mathrm{C} 34$;

skos: notation "15";

skos:prefLabel "Lung"@en.

ecis-code: cancer-site-16 a skos: Concept,

ecis-code: Cancersite;

qb: codelist ecis-code: cancer-site ;

rdfs: isDefinedBy pdf:Estimates_cancer_sites.pdf ;

skos:broader ecis-code: cancer-site-34;

skos: exactMatch icd10:C43;

skos: inscheme ecis-code: cancer-site ;

skos: notation "16" ;

skos:prefLabel "Melanoma"@en.

ecis-code: cancer-site-17 a skos: Concept, ecis-code: Cancersite;

qb: codelist ecis-code: cancer-site ;

rdfs: isDefinedBy pdf: Estimates_cancer_sites.pdf ;

skos:broader ecis-code:cancer-site-34;

skos: exactMatch icd10:c50;

skos: inscheme ecis-code:cancer-site ;

skos: notation "17" ;

skos:prefLabel "Breast"@en.

ecis-code: cancer-site-18 a skos: Concept,

ecis-code: CancerSite;

qb: codelist ecis-code: cancer-site ;

rdfs: isDefinedBy pdf: Estimates_cancer_sites.pdf ;

skos:broader ecis-code:cancer-site-34;

skos: exactMatch icd10:C51;

skos: inscheme ecis-code: cancer-site;

skos: notation "18";

skos: prefLabel "Vulva"@en.

ecis-code: cancer-site-19 a skos: Concept,

ecis-code: CancerSite :

qb: codelist ecis-code: cancer-site ;

rdfs: isDefinedBy pdf:Estimates_cancer_sites.pdf ; skos:broader ecis-code: cancer-site-34;

skos:exactMatch icd10:C52;

skos: inscheme ecis-code: cancer-site;

skos: notation "19" ;

skos: prefLabel "Vagina"@en.

ecis-code: cancer-site-2 a skos: Concept,

ecis-code: Cancersite;

qb: codelist ecis-code: cancer-site ;

rdfs: isDefinedBy pdf:Estimates_cancer_sites.pdf ; skos:broader ecis-code: cancer-site-34;

skos: inscheme ecis-code:cancer-site ;

skos: narrowMatch icd10:c07,

icd10:c08;

skos:notation "2" ;

skos: prefLabel "Salivary glands"@en.

ecis-code: cancer-site-20 a skos: Concept, ecis-code: CancerSite;

qb: codelist ecis-code: cancer-site ;

rdfs: isDefinedBy pdf:Estimates_cancer_sites.pdf ;

skos:broader ecis-code:cancer-site-34;

skos: exactMatch icd10:C53;

skos: inscheme ecis-code: cancer-site;

skos: notation "20" ;

skos: prefLabel "Cervix uteri"@en.

ecis-code: cancer-site-21 a skos: Concept, ecis-code: Cancersite ;

qb: codelist ecis-code: cancer-site ;

rdfs: isDefinedBy pdf:Estimates_cancer_sites.pdf ; skos:broader ecis-code:cancer-site-34;

skos: exactMatch icd10:C54;

skos: inscheme ecis-code:cancer-site ;

skos:notation "21" ;

skos: prefLabel "Corpus uteri"@en.

ecis-code: cancer-site-22 a skos: Concept,

ecis-code: Cancersite;

$\mathrm{qb}$ : codelist ecis-code: cancer-site ;

rdfs: isDefinedBy pdf:Estimates_cancer_sites.pdf ;

skos:broader ecis-code: cancer-site-34;

skos: exactMatch icd10:C56;

skos: inscheme ecis-code:cancer-site ;

skos: notation "22" ;

skos: prefLabel "Ovary"@en.

ecis-code: cancer-site-23 a skos: Concept,

ecis-code: CancerSite;

qb: codelist ecis-code: cancer-site ;

rdfs: isDefinedBy pdf:Estimates_cancer_sites.pdf ;

skos:broader ecis-code: cancer-site-34;

skos: exactMatch icd10:c60;

skos: inscheme ecis-code:cancer-site;

skos: notation "23" ;

skos:prefLabel "Penis"@en.

ecis-code : cancer-site-24 a skos: Concept, ecis-code: CancerSite;

qb: codelist ecis-code: cancer-site;

rdfs: isDefinedBy pdf:Estimates_cancer_sites.pdf ;

skos:broader ecis-code: cancer-site-34;

skos: exactMatch icd10: 61 ;

skos: inscheme ecis-code: cancer-site ;

skos: notation "24" ;

skos: prefLabel "Prostate"@en.

ecis-code: cancer-site-25 a skos: Concept,

ecis-code: Cancersite;

qb: codelist ecis-code: cancer-site ;

rdfs: isDefinedBy pdf:Estimates_cancer_sites.pdf ;

skos:broader ecis-code: cancer-site-34; 


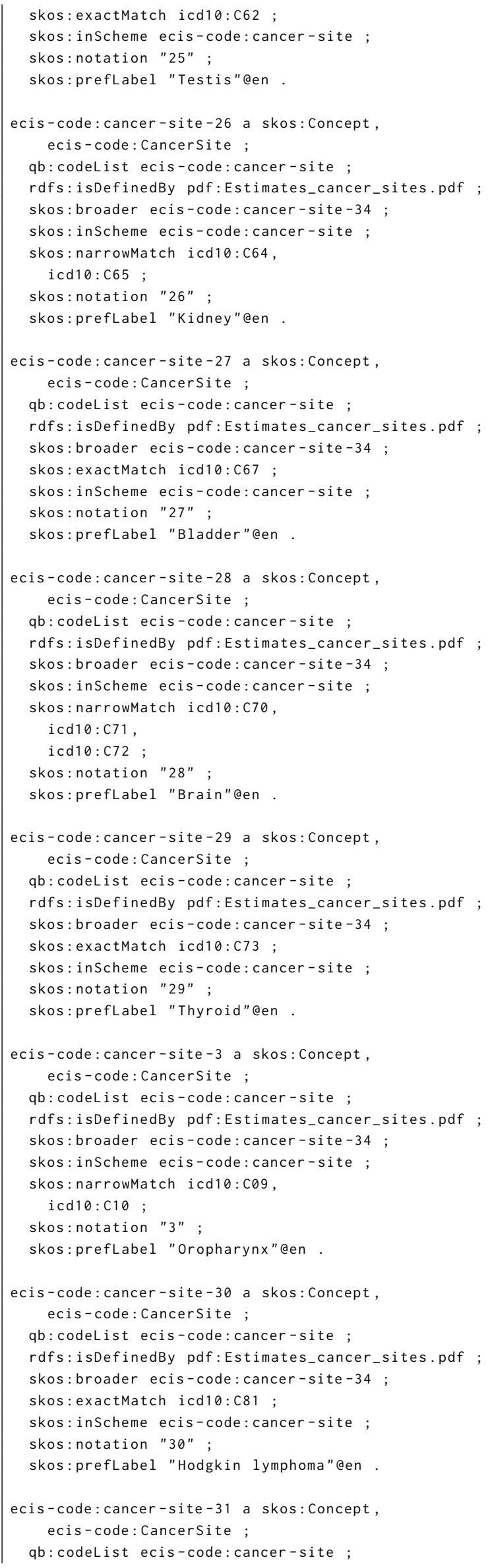

rdfs: isDefinedBy pdf: Estimates_cancer_sites.pdf ; skos:broader ecis-code:cancer-site-34; skos: inscheme ecis-code:cancer-site ; skos: narrowMatch icd10:B21.1,

icd10:B21.2,

icd10:B21.3,

icd10: $\mathrm{C} 82$,

icd10: $\mathrm{c} 83$,

icd10:c84,

icd $10: \mathrm{c} 85$,

icd $10: \mathrm{c} 86$,

$\operatorname{icd} 10: c 88.4$,

icd10:c96;

skos: notation "31";

skos:prefLabel "Non-Hodgkin lymphoma"@en.

ecis-code: cancer-site-32 a skos: Concept,

ecis-code: CancerSite ;

qb: codelist ecis-code: cancer-site ;

rdfs: isDefinedBy pdf: Estimates_cancer_sites.pdf ;

skos:broader ecis-code: cancer-site-34;

skos: inscheme ecis-code:cancer-site ;

skos: narrowMatch icd10: $\mathrm{c} 88$,

icd10:c90;

skos: notation "32" ;

skos:prefLabel "Multiple myeloma"@en.

ecis-code: cancer-site-33 a skos: Concept, ecis-code: Cancersite ;

qb: codelist ecis-code: cancer-site ;

rdfs: isDefinedBy pdf:Estimates_cancer_sites.pdf ; skos:broader ecis-code:cancer-site-34;

skos: inscheme ecis-code:cancer-site ;

skos: narrowMatch icd10:C91,

icd10:c92,

icd10:c93,

icd10:c94,

icd10:c95;

skos: notation "33";

skos:prefLabel "Leukaemia"@en.

ecis-code: cancer-site-4 a skos: Concept,

ecis-code: Cancersite :

qb: codelist ecis-code: cancer-site ;

rdfs: isDefinedBy pdf:Estimates_cancer_sites.pdf ;

skos:broader ecis-code: cancer-site-34;

skos: exactMatch icd10: 111 ;

skos: inscheme ecis-code: cancer-site ;

skos: notation "4" ;

skos:prefLabel "Nasopharynx"@en.

ecis-code: cancer-site-5 a skos: Concept,

ecis-code: CancerSite;

qb: codelist ecis-code: cancer-site ;

rdfs: isDefinedBy pdf:Estimates_cancer_sites.pdf ;

skos:broader ecis-code: cancer-site-34;

skos: inscheme ecis-code: cancer-site ;

skos: narrowMatch icd $10: \mathrm{C} 12$,

icd10: $\mathrm{C} 13$;

skos: notation "5";

skos:prefLabel "Hypopharynx"@en.

ecis-code: cancer-site-6 a skos: Concept,

ecis-code: CancerSite;

qb: codelist ecis-code: cancer-site ;

rdfs: isDefinedBy pdf:Estimates_cancer_sites.pdf ;

skos:broader ecis-code: cancer-site-34;

skos: exactMatch icd10: 15 ;

skos: inscheme ecis-code: cancer-site ;

skos: notation "6";

skos:prefLabel "Oesophagus"@en. 


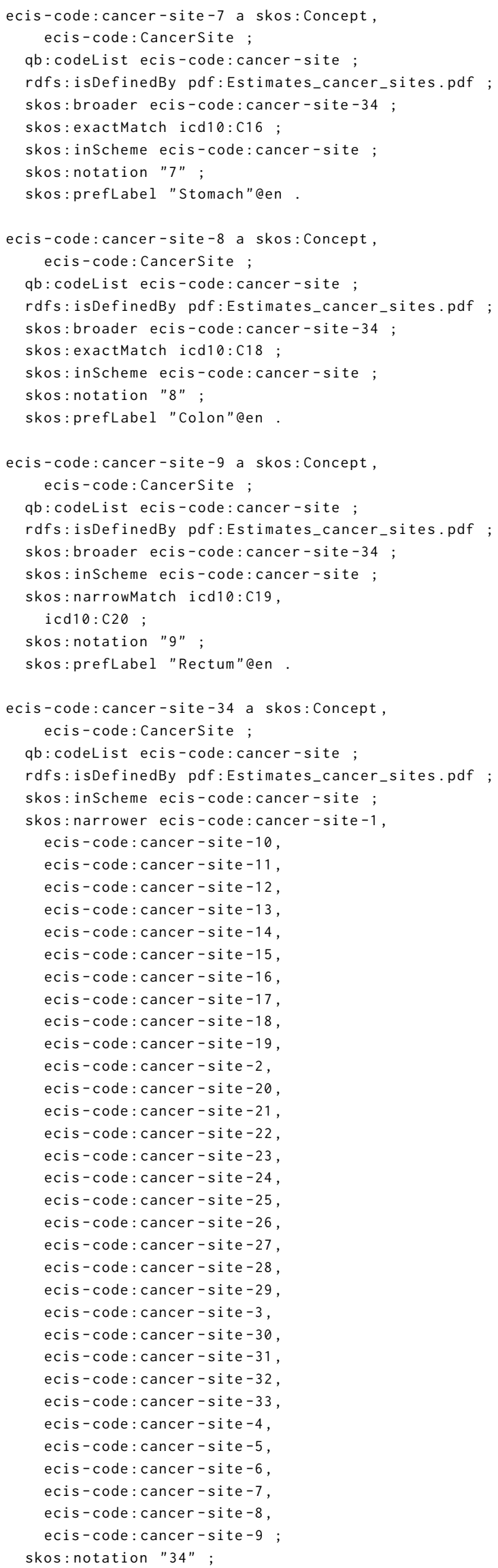

ecis-code: cancer-site a skos: ConceptScheme ; dct:description "List of cancer sites used in ECIS."@en

;

dct:title "ECIS cancer site register"@en ;

rdfs: isDefinedBy pdf:Estimates_cancer_sites.pdf ;

skos: hasTopConcept ecis-code: cancer-site-34.

\section{CRediT authorship contribution statement}

Nicholas Nicholson: Conceptualization, Methodology, Writing - original draft. Andrea Perego: Methodology, Validation, Writing - review \& editing.

\section{References}

[1] Albreht, T., McKee, M., Alexe, D.M., Coleman, M., Martin-Moreno, J., 2008. Making progress against cancer, in: Coleman, M., Alexe, D.M., Albreht, T., McKee, M. (Eds.), Responding to the challenge of cancer in Europe. Institute of Public Health of the Republic of Slovenia, Ljubljana. chapter 16, pp. 315-327. URL: https://apps. who.int/ iris/handle/10665/107879.

[2] Australian Institute of Health and Welfare, 2019. METeOR: Metadata online registry. URL: http://meteor.aihw.gov.au/. (accessed 9 September 2019).

[3] BBMRI-ERIC, 2019. Biobanking and biomolecular resources european research infrastructure (BBMRI-ERIC). URL: http://www. bbmri-eric.eu/. (accessed 9 September 2019).

[4] Bray, T., Paoli, J., Sperberg-McQueen, C.M., Maler, E., Yergeau, F., 2008. Extensible Markup Language (XML) 1.0 (Fifth Edition). W3C Recommendation. World Wide Web Consortium. URL: http://www. W3. org/TR/2008/REC-xml-20081126/.

[5] CHAFEA Health Programmes Database, 2015. Cross-Border Patient Registries Initiative [PARENT] [20112302] - Joint Actions. Project Summary. European Commission. URL: https: //webgate.ec. europa.eu/chafea_pdb/health/projects/20112302/summary/. (accessed 9 September 2019).

[6] CORDIS, 2019a. EUROCOURSE - Europe against Cancer: Optimisation of the Use of Registries for Scientific Excellence in research. Project Fact Sheet. European Commission. URL: https: //cordis. europa. eu/project/id/219453. (accessed 9 September 2019).

[7] CORDIS, 2019b. RD-CONNECT: An integrated platform connecting registries, biobanks and clinical bioinformatics for rare disease research. Project Fact Sheet. European Commission. URL: https: //cordis. europa. eu/project/id/305444. (accessed 9 September 2019).

[8] Cyganiak, R., Reynolds, D., 2014. The RDF Data Cube Vocabulary. W3C Recommendation. World Wide Web Consortium. URL: http: //www.w3.org/TR/2014/REC-vocab-data-cube-20140116/.

[9] ECFS, 2019a. Common data elements metadata, cystic fibrosis. URL: https://www.ecfs.eu/sites/default/files/general-content-files/ working-groups/ecfs-patient-registry/Encounter_Variables_ECFSPR_ V1.2.pdf. (accessed 9 September 2019).

[10] ECFS, 2019b. European cystic fibrosis society patient registry (ECFSPR). URL: https://www.ecfs.eu/projects/ecfs-patient-registry/ project. (accessed 9 September 2019).

[11] eHealth Network, 2015. Recommendations on patients' registries in particular on the use of knowledge gathered through the PARENT Joint Action. Technical Report. European Commission. URL: https://ec. europa.eu/health/sites/health/files/ ehealth/docs/ev_20151123_co05_en.pdf. (accessed 9 September 2019).

[12] EUBIROD Network, 2019. European best information through regional outcomes in diabetes (EUBIROD). URL: http://www. eubirod. eu/. (accessed 9 September 2019).

[13] EUROCISS, 2019a. Common data elements metadata, cardiovascular. URL: http://www. cuore.iss.it/eurociss/reg_ictus/pdf/ictus_ raccolta-dati.pdf. (accessed 9 September 2019). 
[14] EUROCISS, 2019b. European cardiovascular indicators surveillance set (EUROCISS). URL: http://www. cuore. iss.it/eurociss/en/ project/project. asp. (accessed 9 September 2019).

[15] European Commission, 2018. List of cancer sites for estimates 2018 (ECIS). URL: https://ecis.jrc.ec. europa.eu/pdf/Estimates_cancer_ sites. pdf. (accessed 9 September 2019).

[16] European Commission, 2019a. Common data elements metadata, congenital anomalies. URL: https://eu-rd-platform. jrc.ec. europa. eu/sites/default/files/2.2.1b_28_Dec2018.pdf. (accessed 9 September 2019).

[17] European Commission, 2019b. Common data elements metadata, rare diseases. URL: https: //eu-rd-platform. jrc.ec. europa.eu/ set-of-common-data-elements. (accessed 9 September 2019).

[18] European Commission, 2019c. European cancer information system (ECIS). URL: https://ecis.jrc.ec. europa.eu/. (accessed 9 September 2019).

[19] European Commission, 2019d. European platform on rare disease registration (EU RD Platform). URL: https://eu-rd-platform. jrc. ec. europa. eu/. (accessed 9 September 2019).

[20] European Union, 2011. Directive 2011/24/EU of the European Parliament and of the Council of 9 March 2011 on the application of patients' rights in cross-border healthcare. OJ L 88, 45-65. URL: http://data. europa. eu/eli/dir/2011/24/oj.

[21] European Union, 2016. Regulation (EU) 2016/679 of the European Parliament and of the Council of 27 April 2016 on the protection of natural persons with regard to the processing of personal data and on the free movement of such data, and repealing Directive 95/46/EC (General Data Protection Regulation) (Text with EEA relevance). OJ L 119, 1-88. URL: http://data. europa. eu/eli/reg/2016/679/oj.

[22] Fritz, A., Percy, C., Jack, A., et al., 2000. International classification of diseases for oncology, 3rd ed. Technical Report. World Health Organization. URL: https://apps. who. int/iris/handle/10665/42344.

[23] ISO/IEC, 2019. ISO/IEC 11179: Information technology - Metadata Registries (MDR) Parts 1-7. ISO Standards. International Organization for Standardization. URL: http://metadata-standards.org/ $11179 /$.

[24] Klyne, G., Carroll, J.J., McBride, B., 2014. RDF 1.1 Concepts and Abstract Syntax. W3C Recommendation. World Wide Web Consortium. URL: http://www.w3.org/TR/2014/ REC-rdf11-concepts-20140225/.

[25] Lopes, P., Oliveira, J., 2102. COEUS: "semantic web in a box" for biomedical applications. Journal of Biomedical Semantics 3. doi:10. 1186/2041-1480-3-11.

[26] Martos, C., Crocetti, E., Visser, O., Rous, B., Giusti, F., et al., 2018. A proposal on cancer data quality checks: one common procedure for European cancer registries (version 1.1). JRC Technical Report. Publications Office of the European Union. doi:10.2760/429053.

[27] MIABIS Poject, 2019. Minimum information about biobank data sharing (MIABIS) standards. URL: https://github.com/MIABIS/ miabis/wiki. (accessed 9 September 2019).

[28] Miles, A., Bechhofer, S., 2009. SKOS Simple Knowledge Organization System Reference. W3C Recommendation. World Wide Web Consortium. URL: http://www.w3.org/TR/2009/ REC-skos-reference-20090818/.

[29] Nicholson, N., Giusti, F., Martos, C., et al., 2019. An ontology-based approach for developing a harmonised data-validation tool for European cancer registration. Journal of Biomedical Semantics (under review).

[30] Orphanet, 2019. Rare Disease Registries in Europe. Orphanet Report Series. Orphanet. URL: http://www. orpha. net/orphacom/cahiers/ docs/GB/Registries.pdf.

[31] Pajić, V., Čebular, T., Kostešić, M., 2013. Pilot Registry of Registries (RoR) Phase 1 Development Report. Technical Report. PARENT project. URL: https://webgate.ec.europa.eu/chafea_pdb/assets/ files/pdb/20112302/20112302_d06-00_en_ps__pilot_registry_of_ registries_phase_1_development_report.pdf.

[32] Prud'hommeaux, E., Carothers, G., 2014. RDF 1.1 Turtle - Terse RDF Triple Language. W3C Recommendation.
World Wide Web Consortium. URL: http://www.w3.org/TR/2014/ REC-turtle-20140225/.

[33] Reynolds, D., 2013. SKOS Concepts and ConceptSchemes for each COG defined code list. SKOS Vocabulary. UK Government Linked Data Working Group. URL: http://purl.org/linked-data/sdmx/2009/ code\#.

[34] Sernadela, P., González-Castro, L., Carta, C., et al., 2017. Linked registries: Connecting rare diseases patient registries through a semantic web layer. BioMed Research International doi:10.1155/2017/8327980.

[35] Sinaci, A., Laleci Erturkmen, G., 2013. A federated semantic metadata registry framework for enabling interoperability across clinical research and care domains. Journal of Biomedical Informatics 46, 784-794. doi:10.1016/j.jbi.2013.05.009.

[36] Sinaci, A., Laleci Erturkmen, G., Gonul, S., et al., 2015. Postmarketing safety study tool: A web based, dynamic, and interoperable system for postmarketing drug surveillance studies. BioMed Research International doi:10.1155/2015/976272.

[37] SMDX, 2019. Statistical data and metadata exchange (SDMX). URL: https://sdmx.org/. (accessed 18 December 2019).

[38] W3C OWL Working Group, 2012. OWL 2 Web Ontology Language Document Overview (Second Edition). W3C Recommendation. World Wide Web Consortium. URL: http://www.w3.org/TR/ 2012/REC-ow12-overview-20121211/.

[39] W3C SPARQL Working Group, 2013. SPARQL 1.1 Overview. W3C Recommendation. World Wide Web Consortium. URL: http://www. W3. org/TR/2013/REC-sparql11-overview-20130321/.

[40] Wilkinson, M., Dumontier, M., Aalbersberg, I., et al., 2016. The FAIR guiding principles for scientific data management and stewardship. Scientific Data 3. doi:10.1038/sdata.2016.18.

[41] World Health Organization, 2016. International statistical classification of diseases and related health problems - 10th revision (ICD-10 Version:2016). URL: https://icd. who. int/browse10/2016/en.

[42] Zaletel, M., Kralj, M., 2015. Methodological guidelines and recommendations for efficient and rational governance of patient registries. Technical Report. PARENT project. URL: https://ec. europa.eu/health/sites/health/files/ehealth/docs/ patient_registries_guidelines_en.pdf. 